\title{
Practical heteroskedastic Gaussian process modeling for large simulation experiments
}

\author{
Mickaël Binois* $\quad$ Robert B. Gramacy ${ }^{\dagger} \quad$ Mike Ludkovski ${ }^{\ddagger}$
}

\begin{abstract}
We present a unified view of likelihood based Gaussian progress regression for simulation experiments exhibiting input-dependent noise. Replication plays an important role in that context, however previous methods leveraging replicates have either ignored the computational savings that come from such design, or have short-cut full likelihood-based inference to remain tractable. Starting with homoskedastic processes, we show how multiple applications of a well-known Woodbury identity facilitate inference for all parameters under the likelihood (without approximation), bypassing the typical full-data sized calculations. We then borrow a latent-variable idea from machine learning to address heteroskedasticity, adapting it to work within the same thrifty inferential framework, thereby simultaneously leveraging the computational and statistical efficiency of designs with replication. The result is an inferential scheme that can be characterized as single objective function, complete with closed form derivatives, for rapid library-based optimization. Illustrations are provided, including real-world simulation experiments from manufacturing and the management of epidemics.
\end{abstract}

Key words: stochastic kriging, input-dependent noise, Woodbury formula, replication

\section{Introduction}

Simulation-based experimentation has been commonplace in the physical and engineering sciences for decades, and has been advancing rapidly in the social and biological sciences. In both settings, supercomputer resources have dramatically expanded the size of experiments. In the physical/engineering setting, larger experiments are desired to enhance precision and to explore larger parameter spaces. In the social and biological sciences there is a third reason:

\footnotetext{
*Corresponding author: The University of Chicago Booth School of Business, 5807 S. Woodlawn Ave., Chicago IL, 60637; mbinois@chicagobooth.edu

${ }^{\dagger}$ Department of Statistics, Virginia Tech, Hutcheson Hall, 250 Drillfield Drive, Blacksburg, VA 24061

${ }^{\ddagger}$ Department of Statistics and Applied Probability, University of California Santa Barbara, 5520 South Hall Santa Barbara, CA 93106-3110
} 
stochasticity. Whereas in the physical sciences solvers are often deterministic, or if they involve Monte Carlo then the rate of convergence is often known (Picheny and Ginsbourger, 2013), in the social and biological sciences simulations tend to involve randomly interacting agents. In that setting, signal-to-noise ratios can vary dramatically across experiments and for configuration (or input) spaces within experiments. We are motivated by two examples, from inventory control (Hong and Nelson, 2006; Xie et al., 2012) and online management of emerging epidemics ( $\mathrm{Hu}$ and Ludkovski, 2017), which exhibit both features.

Modeling methodology for large simulation efforts with intrinsic stochasticity is lagging. One attractive design tool is replication, i.e., repeated observations at identical inputs. Replication offers a glimpse at pure simulation variance, which is valuable for detecting a weak signal in high noise settings. Replication also holds the potential for computational savings through pre-averaging of repeated observations. It becomes doubly essential when the noise level varies in the input space. Although there are many ways to embellish the classical GP setup for heteroskedastic modeling, e.g., through choices of the covariance kernel, few acknowledge computational considerations. In fact, many exacerbate the problem. A notable exception is stochastic kriging (SK, Ankenman et al., 2010) which leverages replication for thriftier computation in low signal-to-noise regimes, where it is crucial to distinguish intrinsic stochasticity from extrinsic model uncertainty. However, SK has several drawbacks. Inference for unknowns is not based completely on the likelihood. It has the crutch of requiring (a minimal amount of) replication at each design site, which limits its application. Finally, the modeling and extrapolation of input-dependent noise is a secondary consideration, as opposed to one which is fully integrated into the joint inference of all unknowns.

Our contributions address these limitations from both computational and methodological perspectives. On the computational side, we expand upon a so-called Woodbury identity to reduce computational complexity of inference and prediction under replication: from the (log) likelihood of all parameters and its derivatives, to the classical kriging equations. 
We are not the first to utilize the Woodbury "trick" with GPs (see, e.g., Opsomer et al., 1999; Banerjee et al., 2008; $\mathrm{Ng}$ and Yin, 2012), but we believe we are the first to realize its full potential under replication in both homoskedastic and heteroskedastic modeling setups. We provide proofs of new results, and alternate derivations leading to simpler results for quantities first developed in previous papers, including for SK. For example, we establishas identities - results for the efficiency of estimators leveraging replicate-averaged quantities, which previously only held in expectation and under fixed parameterization.

On the methodological end, we further depart from SK and borrow a heteroskedastic modeling idea from the machine learning literature (Goldberg et al., 1998) by constructing an apparatus to jointly infer a spatial field of simulation variances (a noise-field) along with the mean-field GP. The structure of our proposed method is most similar to the one described in Kersting et al. (2007) who turn a "hard expectation maximization (EM)" problem, with expectation replacing the expensive Markov Chain Monte Carlo (MCMC) averaging over latent variables, into a pure maximization problem. Here, we show how the Woodbury trick provides two-fold computational and inferential savings with replicated designs: once for conventional GP calculations arising as a subroutine in the wider heteroskedastic model, and twice by reducing the number of latent variables used to described the noise field. We go on to illustrate how we may obtain inference and prediction in the spirit of SK, but via full likelihood-based inference and without requiring minimal replication at each design site.

A limitation of the Kersting et al. pure maximization approach is a lack of smoothness in the estimated latents. We therefore propose to interject an explicit smoothing back into the objective function being optimized. This smoother derives from a GP prior on the latent (log) variance process, and allows one to explicitly model and optimize the spatial correlation of the intrinsic stochasticity. It serves to integrate noise-field and mean-field in contrast to the SK approach of separate empirical variance calculations on replicates, followed by independent auxiliary smoothing for out-of-sample prediction. We implement 
the smoothing in such a way that full derivatives are still available, for the latents as well as the coupled GP hyperparameters, so that the whole inferential problem can be solved by deploying an off-the-shelf library routine. Crucially, our smoothing mechanism does not bias the predictor at the stationary point solving the likelihood equations, compared to an un-smoothed alternative. Rather, it has an annealing effect on the likelihood, and makes the solver easier to initialize, both accelerating its convergence.

The remainder of the paper is organized as follows. In Section 2 we review relevant GP details, SK, and latent noise variables. Section 3 outlines the Woodbury trick for decomposing the covariance when replication is present, with application to inference and prediction schemes under the GP. Practical heteroskedastic modeling is introduced in Section 4, with the Woodbury decomposition offering the double benefit of fewer latent variables and faster matrix decompositions. An empirical comparison on a cascade of alternatives, on toy and real data, is entertained in Section 5. We conclude with a brief discussion in Section 6 .

\section{GPs under replication and heteroskedasticity}

Standard kriging builds a surrogate model of an unknown function $f: \mathbb{R}^{d} \rightarrow \mathbb{R}$ given a set of noisy output observations $\mathbf{Y}=\left(y_{1}, \ldots, y_{N}\right)^{\top}$ at design locations $\mathbf{X}=\left(\mathbf{x}_{1}, \ldots, \mathbf{x}_{N}\right)^{\top}$. This is achieved by putting a so-called Gaussian process (GP) prior on $f$, characterized by a mean function and covariance function $k: \mathbb{R}^{d} \times \mathbb{R}^{d} \rightarrow \mathbb{R}$. We follow the simplifying assumption in the computer experiments literature in using a mean zero GP, which shifts all of the modeling effort to the covariance structure. The covariance or kernel $k$ is positive definite, with parameterized families such as the Gaussian or Matérn being typical choices.

Observation model is $y\left(\mathbf{x}_{i}\right)=f\left(\mathbf{x}_{i}\right)+\varepsilon_{i}, \varepsilon_{i} \sim \mathcal{N}\left(0, r\left(\mathbf{x}_{i}\right)\right)$. In the typical homoskedastic case $r(\mathbf{x})=\tau^{2}$ is constant, but we anticipate heteroskedastic models as well. In this setup, the modeling framework just described is equivalent to writing $Y \sim \mathcal{N}_{N}\left(\mathbf{0}, \mathbf{K}_{N}+\boldsymbol{\Sigma}_{N}\right)$, where 
$\mathbf{K}_{N}$ is the $N \times N$ matrix with $i j$ coordinates $k\left(\mathbf{x}_{i}, \mathbf{x}_{j}\right)$, and $\boldsymbol{\Sigma}_{N}=\operatorname{Diag}\left(r\left(\mathbf{x}_{1}\right), \ldots, r\left(\mathbf{x}_{N}\right)\right)$ is the noise matrix. Notice that $\mathbf{x}_{i}$ is a $d \times 1$ vector and the $\varepsilon_{i}$ 's are i.i.d..

Given a form for $k(\cdot, \cdot)$, multivariate normal (MVN) conditional identities provide a predictive distribution at site $\mathbf{x}: Y(\mathbf{x}) \mid \mathbf{Y}$, which is Gaussian with parameters

$$
\begin{aligned}
\mu(\mathbf{x}) & =\mathbb{E}(Y(\mathbf{x}) \mid \mathbf{Y})=\mathbf{k}(\mathbf{x})^{\top}\left(\mathbf{K}_{N}+\mathbf{\Sigma}_{N}\right)^{-1} \mathbf{Y}, \text { where } \mathbf{k}(\mathbf{x})=\left(k\left(\mathbf{x}, \mathbf{x}_{1}\right), \ldots, k\left(\mathbf{x}, \mathbf{x}_{N}\right)\right)^{\top} \\
\sigma^{2}(\mathbf{x}) & =\operatorname{Var}(Y(\mathbf{x}) \mid \mathbf{Y})=k(\mathbf{x}, \mathbf{x})+r(\mathbf{x})-\mathbf{k}(\mathbf{x})^{\top}\left(\mathbf{K}_{N}+\mathbf{\Sigma}_{N}\right)^{-1} \mathbf{k}(\mathbf{x}) .
\end{aligned}
$$

The kernel $k$ has hyperparameters that must be estimated. Among a variety of methods, many are based on the likelihood, which is simply a MVN density. Most applications involve stationary kernels, $k\left(\mathbf{x}, \mathbf{x}^{\prime}\right)=\nu c\left(\mathbf{x}-\mathbf{x}^{\prime} ; \boldsymbol{\theta}\right)$, i.e., $\mathbf{K}_{N}=\nu \mathbf{C}_{N}$, with $\nu$ being the process variance and $\boldsymbol{\theta}$ being additional hyperparameters of the correlation function $c$. After relabeling $\mathbf{K}_{N}+$ $\Sigma_{N}=\nu\left(\mathbf{C}_{N}+\boldsymbol{\Lambda}_{N}\right)$, first order optimality conditions provide a plug-in estimator for the common factor $\nu: \hat{\nu}=N^{-1} \mathbf{Y}^{\top}\left(\mathbf{C}_{N}+\boldsymbol{\Lambda}_{N}\right)^{-1} \mathbf{Y}$. The log-likelihood conditional on $\hat{\nu}$ is then:

$$
\log L=-\frac{N}{2} \log (2 \pi)-\frac{N}{2} \log \hat{\nu}-\frac{1}{2} \log \left|\mathbf{C}_{N}+\boldsymbol{\Lambda}_{N}\right|-\frac{N}{2} .
$$

Observe that we must decompose (e.g., via Cholesky) $\mathbf{C}_{N}+\boldsymbol{\Lambda}_{N}$ potentially multiple times in a maximization scheme, for inversion and determinant computations, which requires $\mathcal{O}\left(N^{3}\right)$ operations for the typical choices of $c$. This cubic computation severely limits the experiment size, $N$, that can be modeled with GPs. Some work-arounds for large data include approximate strategies (e.g., Banerjee et al., 2008; Haaland and Qian, 2011; Kaufman et al., 2012, Eidsvik et al., 2014; Gramacy and Apley, 2015) or a degree of tailoring to the simulation mechanism or the input design (Plumlee, 2014, Nychka et al., 2015).

Replicates: When replication is present, some of the design sites are repeated. This offers a potential computational advantage via switching from the full- $N$ size of the data to 
the unique- $n$ number of unique design locations. To make this precise, we introduce a special notation; note that our setting is fully generic and nests the standard setting by allowing the number of replicates to vary site-to-site, or be absent altogether.

Let $\overline{\mathbf{x}}_{i}, 1=i, \ldots, n$ represent the $n \ll N$ unique input locations, and $y_{i}^{(j)}$ be the $j^{\text {th }}$ out of $a_{i} \geq 1$ replicates, i.e., $j=1, \ldots, a_{i}$, observed at $\overline{\mathbf{x}}_{i}$, where $\sum_{i=1}^{n} a_{i}=N$. Then let $\overline{\mathbf{Y}}=\left(\bar{y}_{1}, \ldots, \bar{y}_{n}\right)^{\top}$ collect averages of replicates, $\bar{y}_{i}=\frac{1}{a_{i}} \sum_{j=1}^{a_{i}} y_{i}^{(j)}$. We now develop a map from full $\mathbf{K}_{N}, \boldsymbol{\Sigma}_{N}$ matrices to their unique- $n$ counterparts. Without loss of generality, assume that the data are ordered so that $\mathbf{X}=\left(\overline{\mathbf{x}}_{1}, \ldots, \overline{\mathbf{x}}_{1}, \ldots \overline{\mathbf{x}}_{n}\right)^{\top}$ where each input is repeated $a_{i}$ times, and where $\mathbf{Y}$ is stacked with observations on the $a_{i}$ replicates in the same order. With $\mathbf{X}$ composed in this way, we have $\mathbf{X}=\mathbf{U} \overline{\mathbf{X}}$, with $\mathbf{U}$ the $N \times n$ block matrix $\mathbf{U}=\operatorname{Diag}\left(\mathbf{1}_{a_{1}, 1}, \ldots, \mathbf{1}_{a_{n}, 1}\right)$, where $\mathbf{1}_{k, l}$ is $k \times l$ matrix filled with ones. Similarly, $\mathbf{K}_{N}=\mathbf{U K}_{n} \mathbf{U}^{\top}$, where $\mathbf{K}_{n}=\left(k\left(\overline{\mathbf{x}}_{i}, \overline{\mathbf{x}}_{j}\right)\right)_{1 \leq i, j, \leq n}$ while $\mathbf{U}^{\top} \boldsymbol{\Sigma}_{N} \mathbf{U}=\mathbf{A}_{n} \boldsymbol{\Sigma}_{n}$ where $\boldsymbol{\Sigma}_{n}=\operatorname{Diag}\left(r\left(\overline{\mathbf{x}}_{1}\right), \ldots, r\left(\overline{\mathbf{x}}_{n}\right)\right)$ and $\mathbf{A}_{n}=\operatorname{Diag}\left(a_{1}, \ldots, a_{n}\right)$. This decomposition is the basis for the Woodbury identities exploited in Section 3. Henceforth, we utilize this notation with $n$ and $N$ subscripts highlighting the size of matrices and vectors.

\subsection{Stochastic kriging}

A hint for the potential gains available with replication, while addressing heteroskedasticity, appears in Ankenman et al. (2010) who show that the unique- $n$ predictive equations

$$
\begin{aligned}
& \mu_{n}(\mathbf{x})=\mathbf{k}_{n}(\mathbf{x})^{\top}\left(\mathbf{K}_{n}+\mathbf{A}_{n}^{-1} \boldsymbol{\Sigma}_{n}\right)^{-1} \overline{\mathbf{Y}} \quad \text { where } \quad \mathbf{k}_{n}(\mathbf{x})=\left(k\left(\mathbf{x}, \overline{\mathbf{x}}_{1}\right), \ldots, k\left(\mathbf{x}, \overline{\mathbf{x}}_{n}\right)\right)^{\top}, \\
& \sigma_{n}^{2}(\mathbf{x})=k(\mathbf{x}, \mathbf{x})+r(\mathbf{x})-\mathbf{k}_{n}(\mathbf{x})^{\top}\left(\mathbf{K}_{n}+\mathbf{A}_{n}^{-1} \boldsymbol{\Sigma}_{n}\right)^{-1} \mathbf{k}_{n}(\mathbf{x})
\end{aligned}
$$

are unbiased and minimize mean-squared prediction error. This result implies that one can handle $N \gg n$ points in $\mathcal{O}\left(n^{3}\right)$ time. A snag is that one can rarely presume to know the 
variance function $r(\mathbf{x})$. Ankenman et al., however show that with a sensible estimate

$$
\widehat{\boldsymbol{\Sigma}}_{n}=\operatorname{Diag}\left(\hat{\sigma}_{1}^{2}, \ldots, \hat{\sigma}_{n}^{2}\right), \quad \text { where } \quad \hat{\sigma}_{i}^{2}=\frac{1}{a_{i}-1} \sum_{j=1}^{a_{i}}\left(y_{i}^{(j)}-\bar{y}_{i}\right)^{2}
$$

in place of $\boldsymbol{\Sigma}_{n}$ the resulting $\mu_{n}(\mathbf{x})$ is still unbiased with $a_{i} \gg 1$ (they recommend $a_{i} \geq 10$ ). The predictive variance $\sigma_{n}^{2}$ still requires an $r(\mathbf{x})$, for which no observations are directly available for estimation. One could specify $r(\mathbf{x})=0$ and be satisfied with an estimate of extrinsic variance, i.e., filtering heterogeneous noise (Roustant et al., 2012), however that would preclude any applications requiring full uncertainty quantification. Alternatively, Ankenman et al., proposed a second, separately estimated, (no-noise) GP prior for $r$ trained on the $\left(\overline{\mathbf{x}}_{i}, \hat{\sigma}_{i}^{2}\right)$ pairs to obtain a prediction for $r(\mathbf{x})$ at new $\mathbf{x}$ locations.

Although this approach has much to recommend it, there are several notable shortcomings. One is the difference of treatment between hyperparameters. The noise variances are obtained from the empirical variance, while any other parameter, such as lengthscales $\boldsymbol{\theta}$ for $k(\cdot, \cdot)$, are inferred based on the pre-averaged $\log$-likelihood (requiring $\mathcal{O}\left(n^{3}\right)$ for evaluation):

$$
\log \bar{L}:=-\frac{n}{2} \log (2 \pi)-\frac{1}{2} \log \left|\mathbf{K}_{n}+\mathbf{A}_{n}^{-1} \widehat{\boldsymbol{\Sigma}}_{n}\right|-\frac{1}{2} \overline{\mathbf{Y}}^{\top}\left(\mathbf{K}_{n}+\mathbf{A}_{n}^{-1} \widehat{\boldsymbol{\Sigma}}_{n}\right)^{-1} \overline{\mathbf{Y}}
$$

While logical and thrifty, this choice of $\widehat{\boldsymbol{\Sigma}}_{n}$ leads to the requirement of a minimal number of replicates, $a_{i}>1$ for all $i$, meaning that incorporating even a single observation without a second replicate requires a new method (lest such valuable observation(s) be dropped from the analysis). Finally, the separate modeling of the mean-field $f(\cdot)$ and its variance $r(\cdot)$ is inefficient. Ideally, a likelihood would be developed for all unknowns jointly.

\subsection{Latent variable process}

Goldberg et al. (1998) were the first to propose a joint model for $f$ and $r$, coupling a GP 
on the mean with GP on latent log variance (to ensure positivity) variables. Although ideal from a modeling perspective, and not requiring replicated data, inference for the unknowns involved a cumbersome MCMC. Since each MCMC iteration involves iterating over each of $\mathcal{O}(N)$ parameters, with acceptance criteria requiring $\mathcal{O}\left(N^{3}\right)$ calculation, the method was effectively $\mathcal{O}\left(T N^{4}\right)$ to obtain $T$ samples, a severely limiting cost.

Several groups of authors subsequently introduced thriftier alternatives in a similar spirit, essentially replacing MCMC with maximization (Kersting et al., 2007; Quadrianto et al., 2009: Lazaro-Gredilla and Titsias, 2011), but the overall computational complexity of each iteration of search remained the same. An exception is the technique of Boukouvalas and Cornford (2009) who expanded on the EM-inspired method of Kersting et al. (2007) to exploit computational savings that comes from replication in the design. However that framework has two drawbacks. One is that their mechanism for leveraging of replicates unnecessarily, as we show, approximates predictive and inferential quantities compared to full data analogues. Another is that, although the method is inspired by EM, the latent log variances are maximized rather than averaged over, yielding a solution which is not a smooth realization from a GP. Quadrianto et al. (2009) addressed that lack of smoothness by introducing a penalization in the likelihood, but required a much harder optimization.

In what follows we ultimately borrow the modeling apparatus of Goldberg et al. and the EM-inspired method of Kersting et al. and Quadrianto et al., but there are two important and novel ingredients that are crucial to the adhesive binding them together. We show that the Woodbury trick, when fully developed below, facilitates massive computational savings when there is large-scale replication, achieving the same computational order as Boukouvalas and Cornford and SK but without approximation. We then introduce a smoothing step that achieves an EM-style averaging over latent variance, yet the overall method remains in a pure maximizing framework requiring no simulation or otherwise numerical integration. Our goal is to show that it is possible to get the best of all worlds: fully likelihood-based smoothed and 
joint inference of the latent variances alongside the mean-field (mimicking Goldberg et al. but with the $O\left(n^{3}\right)$ computational demands of SK). The only downside is that our point estimates do not convey the same full posterior uncertainty as an MCMC would.

\section{Fast GP inference and prediction under replication}

We exploit the structure of replication in design for GPs with extensive use of two well-known formulas, together comprising the Woodbury identity (e.g., Harville, 1997):

$$
\begin{aligned}
(\mathbf{D}+\mathbf{U B V})^{-1} & =\mathbf{D}^{-1}-\mathbf{D}^{-1} \mathbf{U}\left(\mathbf{B}^{-1}+\mathbf{V D}^{-1} \mathbf{U}\right)^{-1} \mathbf{V D}^{-1} \\
|\mathbf{D}+\mathbf{U B V}| & =\left|\mathbf{B}^{-1}+\mathbf{V D}^{-1} \mathbf{U}\right| \times|\mathbf{B}| \times|\mathbf{D}|
\end{aligned}
$$

where $\mathbf{D}$ and $\mathbf{B}$ are invertible matrices of size $N \times N$ and $n \times n$ respectively, and $\mathbf{U}$ and $\mathbf{V}^{\top}$ are of size $N \times n$. In our context of GP prediction and inference, under the generic covariance parameterization $\mathbf{K}_{N}+\boldsymbol{\Sigma}_{N}=\nu\left(\mathbf{C}_{N}+\boldsymbol{\Lambda}_{N}\right)$, we take the matrix $\mathbf{D}=\boldsymbol{\Sigma}_{N}=\nu \boldsymbol{\Lambda}_{N}$ in (4) as diagonal, e.g., $\nu \boldsymbol{\Lambda}_{N}=\tau^{2} \mathbf{I}_{N}$, and $\mathbf{V}=\mathbf{U}^{\top}$. Moreover, $\boldsymbol{\Sigma}_{N}$ shares the low dimensional structure, $\mathbf{U}^{\top} \boldsymbol{\Sigma}_{N} \mathbf{U}=\mathbf{A}_{n} \boldsymbol{\Sigma}_{n}=\nu \mathbf{A}_{n} \boldsymbol{\Lambda}_{n}$, and note that $\mathbf{U}^{\top} \mathbf{U}=\mathbf{A}_{n}$. In combination, these observations allow efficient computation of the inverse and determinant of $\left(\mathbf{K}_{N}+\boldsymbol{\Sigma}_{N}\right)$. In fact, there is no need to ever build the full- $N$ matrices.

Lemma 3.1. We have the following full- $N$ to unique-n identities for the GP prediction equations, conditional on hyperparameters.

$$
\begin{gathered}
\mathbf{k}_{N}(\mathbf{x})^{\top}\left(\mathbf{K}_{N}+\mathbf{\Sigma}_{N}\right)^{-1} \mathbf{Y}=\mathbf{c}_{n}(\mathbf{x})^{\top}\left(\mathbf{C}_{n}+\boldsymbol{\Lambda}_{n} \mathbf{A}_{n}^{-1}\right)^{-1} \overline{\mathbf{Y}} \\
\mathbf{k}_{N}(\mathbf{x})^{\top}\left(\mathbf{K}_{N}+\boldsymbol{\Sigma}_{N}\right)^{-1} \mathbf{k}_{N}(\mathbf{x})=\nu \mathbf{c}_{n}(\mathbf{x})^{\top}\left(\mathbf{C}_{n}+\boldsymbol{\Lambda}_{n} \mathbf{A}_{n}^{-1}\right)^{-1} \mathbf{c}_{n}(\mathbf{x}) .
\end{gathered}
$$

Eq. (6) establishes that the GP predictive mean, calculated on the average responses at 
replicates and with covariances calculated only at the unique design sites, is indeed identical to the original predictive equations built by overlooking the structure of replication. Eq. (7) reveals the same result for the predictive variance. A proof of this lemma is in Appendix A.1. These identities support common practice, especially in the case of the predictive mean, of only utilizing the $n$ observations in $\overline{\mathbf{Y}}$ for prediction. Ankenman et al. (2010), for example, show that this unique- $n$ shortcut, applied via SK with $\boldsymbol{\Lambda}_{n}=\nu^{-1} \widehat{\boldsymbol{\Sigma}}_{n}$ is the best linear unbiased predictor (BLUP), after conditioning on the hyperparameters in the system. This is simply a consequence of the unique- $n$ predictive equations being identical to their full- $N$ counterpart, inheriting BLUP and any other properties.

Although aspects of the results above have been known for some time, if perhaps not directly connected to the Woodbury formula, they were not (to our knowledge) known to extend to the full likelihood. The lemma below establishes that indeed they do.

Lemma 3.2. Let $\boldsymbol{\Upsilon}_{n}:=\mathbf{C}_{n}+\mathbf{A}_{n}^{-1} \boldsymbol{\Lambda}_{n}$. Then we have the following unique-n identity for the full-N expression for the conditional log likelihood, $\log L$ in Eq. (1).

$$
\begin{aligned}
\log L & =\text { Const }-\frac{N}{2} \log \hat{\nu}_{N}-\frac{1}{2} \sum_{i=1}^{n}\left[\left(a_{i}-1\right) \log \lambda_{i}+\log a_{i}\right]-\frac{1}{2} \log \left|\mathbf{\Upsilon}_{n}\right|, \\
\text { where } \quad \hat{\nu}_{N} & :=N^{-1}\left(\mathbf{Y}^{\top} \boldsymbol{\Lambda}_{N}^{-1} \mathbf{Y}-\overline{\mathbf{Y}}^{\top} \mathbf{A}_{n} \boldsymbol{\Lambda}_{n}^{-1} \overline{\mathbf{Y}}+\overline{\mathbf{Y}}^{\top} \mathbf{\Upsilon}_{n}^{-1} \overline{\mathbf{Y}}\right)
\end{aligned}
$$

The proof of the lemma is based on the following key computations:

$$
\begin{aligned}
\mathbf{Y}^{\top}\left(\mathbf{C}_{N}+\boldsymbol{\Lambda}_{N}\right)^{-1} \mathbf{Y} & =\mathbf{Y}^{\top} \boldsymbol{\Lambda}_{N}^{-1} \mathbf{Y}-\overline{\mathbf{Y}}^{\top} \mathbf{A}_{n} \boldsymbol{\Lambda}_{n}^{-1} \overline{\mathbf{Y}}+\overline{\mathbf{Y}}^{\top}\left(\mathbf{C}_{n}+\mathbf{A}_{n}^{-1} \boldsymbol{\Lambda}_{n}\right)^{-1} \overline{\mathbf{Y}} \\
\log \left|\mathbf{C}_{N}+\boldsymbol{\Lambda}_{N}\right| & =\log \left|\mathbf{C}_{n}+\mathbf{A}_{n}^{-1} \boldsymbol{\Lambda}_{n}\right|+\sum_{i=1}^{n}\left[\left(a_{i}-1\right) \log \lambda_{i}+\log a_{i}\right] .
\end{aligned}
$$

Assuming the $n \times n$ matrices have already been decomposed, at $\mathcal{O}\left(n^{3}\right)$ cost, the extra computational complexity is $\mathcal{O}(N+n)$ for the right-hand-side of $(10)$ and $\mathcal{O}(n)$ for the righthand-side of (11), respectively. Both are negligible compared to $O\left(n^{3}\right)$. 
It is instructive to compare (8 9) to the pre-averaged log likelihood (3) used by SK. In particular, observe that the expression in (9) is different to the one that would be obtained by estimating $\nu$ with unique- $n$ calculations based on $\overline{\mathbf{Y}}$, which would give $\hat{\nu}_{n}=n^{-1} \overline{\mathbf{Y}}^{\top} \mathbf{\Upsilon}_{n}^{-1} \overline{\mathbf{Y}}$, an $\mathcal{O}\left(n^{2}\right)$ calculation assuming pre-decomposed matrices. However, our full data calculation via $\mathbf{Y}$ above gives $\hat{\nu}_{N}=N^{-1}\left(\mathbf{Y}^{\top} \boldsymbol{\Lambda}_{N}^{-1} \mathbf{Y}-\overline{\mathbf{Y}}^{\top} \mathbf{A}_{n} \boldsymbol{\Lambda}_{n}^{-1} \overline{\mathbf{Y}}+n \hat{\nu}_{n}\right)$. The extra term in front of $\hat{\nu}_{n}$ is an important correction for the variance at replicates:

$$
N^{-1}\left(\mathbf{Y}^{\top} \boldsymbol{\Lambda}_{N}^{-1} \mathbf{Y}-\overline{\mathbf{Y}} \mathbf{A}_{n} \boldsymbol{\Lambda}_{n}^{-1} \overline{\mathbf{Y}}\right)=N^{-1} \sum_{i=1}^{n} \frac{a_{i}}{\lambda_{i}} s_{i}^{2}
$$

where $s_{i}^{2}=\frac{1}{a_{i}} \sum_{j=1}^{a_{i}}\left(y_{i}^{(j)}-\bar{y}_{i}\right)^{2}$, i.e., the bias un-adjusted estimate of $\operatorname{Var}\left(Y\left(\mathbf{x}_{i}\right)\right)$ based on $\left\{y_{i}^{(j)}\right\}_{j=1}^{a_{i}}$. Therefore, observe that as the $a_{i}$ get large, Eq. $12 p$ converges to $N^{-1} \sum_{i=1}^{n} \frac{a_{i}}{\lambda_{i}} \operatorname{Var}\left(Y\left(\mathbf{x}_{i}\right)\right)$. Note that computing (12) is in $\mathcal{O}\left(N+n^{2}\right)$ with pre-decomposed matrices.

Finally, we provide the derivative of the unique- $n \log$ likelihood to aid in numerical optimization, revealing a computational complexity in $\mathcal{O}\left(N+n^{3}\right)$, or essentially $\mathcal{O}\left(n^{3}\right)$ :

$$
\begin{aligned}
\frac{\partial \log L}{\partial \cdot} & =\frac{N}{2} \frac{\partial\left(\mathbf{Y}^{\top} \boldsymbol{\Lambda}_{N}^{-1} \mathbf{Y}-\overline{\mathbf{Y}} \mathbf{A}_{n} \boldsymbol{\Lambda}_{n}^{-1} \overline{\mathbf{Y}}+n \hat{\nu}_{n}\right)}{\partial \cdot} \times\left(N \hat{\nu}_{N}\right)^{-1} \\
& -\frac{1}{2} \sum_{i=1}^{n}\left[\left(a_{i}-1\right) \frac{\partial \log \lambda_{i}}{\partial \cdot}\right]-\frac{1}{2} \operatorname{tr}\left(\boldsymbol{\Upsilon}_{n}^{-1} \frac{\partial \mathbf{\Upsilon}_{n}}{\partial \cdot}\right) .
\end{aligned}
$$

The calculations above are presented generically so that they can be applied both in homoskedastic and heteroskedastic settings. In a homoskedastic setting, recall that we may take $\lambda_{i}:=\lambda=\tau^{2} / \nu$, and no further modifications are necessary provided that we choose a form for the covariance structure, generating $\mathbf{C}_{n}$ above, which can be differentiated in closed form. In the heteroskedastic case, described in detail below, we propose a scheme for estimating the latent $\lambda_{i}$ value at the $i^{\text {th }}$ unique design location $\overline{\mathbf{x}}_{i}$. Appendix A.2 provides an empirical illustration of the computational savings that comes from utilizing these identities in a homoskedastic context, and in the presence a modest degree of replication(3). 


\section{$4 \quad$ Practical heteroskedastic modeling}

Heteroskedastic GP modeling involves learning the diagonal matrix $\boldsymbol{\Lambda}_{N}$ (or its unique- $n$ counterpart $\boldsymbol{\Lambda}_{n}$ ), allowing the $\lambda_{i}$ values to exhibit heterogeneity, i.e., not all $\lambda_{i}=\tau^{2} / \nu$ as in the homoskedastic case. Care is required when performing inference for such a high dimensional parameter. The strategy of Goldberg et al. (1998) involves applying regularization in the form of a prior that enforces positivity and encourages a smooth evolution over the input space. Inference is performed by integrating over the posterior of these latent values with MCMC. This is a monumental task when $N$ is large, primarily because mixing of the latents is poor. We show that it is possible to have far fewer latent variables via $\boldsymbol{\Lambda}_{n}$ (recall that $\mathbf{U}^{\top} \boldsymbol{\Lambda}_{N} \mathbf{U}=\mathbf{A}_{n} \boldsymbol{\Lambda}_{n}$ ) when replication is present, via the Woodbury identities above, and to achieve the effect of integration by maximizing a criterion involving smoothed quantities.

\subsection{A new latent process formulation}

A straightforward approach to learning $\boldsymbol{\Lambda}_{n}$ comes by maximizing the log likelihood (8). As we show later in Figure 1 (top), this results in over-fit. As in many high-dimensional settings, it helps to regularize. Toward that end Goldberg et al. suggest a GP prior for $\log \lambda$. This constrains the $\log \boldsymbol{\Lambda}_{n}$ to follow a MVN law, but otherwise they are free to take on any values. In particular, if the MVN acknowledges that our glimpse at the noise process, via the observed $(x, y)$-values, is itself noisy, then the $\hat{\lambda}_{i}$ s that come out, as may be obtained from a local maximum of the resulting penalized likelihood (i.e., the joint posterior for mean-field and noise processes), may not be smooth. Guaranteeing smoothed values via maximizing, as an alternative to more expensive numerical integration, requires an explicit smoothing maneuver in the "objective" used to solve for latents and hyperparameters.

Toward that end we re-cast the $\lambda_{1}, \ldots, \lambda_{n}$ of $\boldsymbol{\Lambda}_{n}$ as derived quantities obtained via the predictive mean of a regularizing GP on new latent variables $\delta_{1}, \ldots, \delta_{n}$, stored in diagonal 
$\boldsymbol{\Delta}_{n}$ for ease of manipulation. That is

$$
\log \boldsymbol{\Lambda}_{n}=\mathbf{C}_{(g)}\left(\mathbf{C}_{(g)}+g \mathbf{A}_{n}^{-1}\right)^{-1} \boldsymbol{\Delta}_{n}=: \mathbf{C}_{(g)} \mathbf{\Upsilon}_{(g)}^{-1} \boldsymbol{\Delta}_{n}
$$

where $\mathbf{C}_{(g)}$ generically denotes the correlation structure of this noise process, whose nugget is $g$, and $\boldsymbol{\Delta}_{n} \sim \mathcal{N}_{n}\left(0, \nu_{(g)}\left(\mathbf{C}_{(g)}+g \mathbf{A}_{n}^{-1}\right)\right)$. The appearance of the replication counts $\mathbf{A}_{n}$ in $\boldsymbol{\Upsilon}_{(g)}=\left(\mathbf{C}_{(g)}+g \mathbf{A}_{n}^{-1}\right)$ is matching the structure of $\boldsymbol{\Upsilon}_{n}$ in Section 3; see Lemma 3.2 or Eq. (10). Thus the right-hand-side of (14) can be viewed as a smoother of the latent $\delta_{i}$ 's, carried out in analogue to the GP equations for the mean-field. This setup guarantees that every $\boldsymbol{\Delta}_{n}$, e.g., as considered by a numerical solver maximizing a likelihood, leads to smooth and positive variances $\boldsymbol{\Lambda}_{n}$. Although any smoother could be used to convert latent $\boldsymbol{\Delta}_{n}$ into $\Lambda_{n}$, a GP mimics the choice of post-hoc smoother in SK. The difference here is that the inferential stage acknowledges a desire for smoothed predictions too.

Choosing a GP smoother has important implications for the stationary point of the composite likelihood developed in Section 4.2 below, positioning smoothing as a computational device that facilitates automatic initialization of the solver and an annealing of the objective for improved numerical stability. The parameter $g$ governs the degree of smoothing: when $g=0$ we have $\log \boldsymbol{\Lambda}_{n}=\boldsymbol{\Delta}_{n}$, i.e., no smoothing; alternatively if $\mathbf{C}_{(g)}=\mathbf{I}_{n}$ and $\boldsymbol{\Delta}_{n}=\mathbf{I}_{n}$, then $\log \boldsymbol{\Lambda}_{n}=(g+1)^{-1} \mathbf{I}_{n}$ and we recover the homoskedastic setup of Section 1 with noise variance $\tau^{2}=\hat{\nu}_{N} \exp (1 /(g+1))$.

To learn appropriate latent $\boldsymbol{\Delta}_{n}$ values (leaving details for other hyperparameters to Section 4.2, we take advantage of a simple chain rule for the log-likelihood (13).

$$
\begin{gathered}
\frac{\partial \log L}{\partial \boldsymbol{\Delta}_{n}}=\frac{\partial \boldsymbol{\Lambda}_{n}}{\partial \boldsymbol{\Delta}_{n}} \frac{\partial \log L}{\partial \boldsymbol{\Lambda}_{n}}=\boldsymbol{\Lambda}_{n} \mathbf{C}_{(g)} \boldsymbol{\Upsilon}_{(g)}^{-1} \frac{\partial \log L}{\partial \boldsymbol{\Lambda}_{n}} \\
\text { where } \quad \frac{\partial \log L}{\partial \lambda_{i}}=\frac{N}{2} \times \frac{\frac{a_{i} s_{i}^{2}}{\lambda_{i}^{2}}+\frac{\left(\boldsymbol{\Upsilon}_{n}^{-1} \overline{\mathbf{Y}}\right)_{i}^{2}}{a_{i}}}{\hat{\nu}_{N}}-\frac{a_{i}-1}{2 \lambda_{i}}-\frac{1}{2 a_{i}}\left(\boldsymbol{\Upsilon}_{n}\right)_{i, i}^{-1} .
\end{gathered}
$$


With $\mathbf{S}=\operatorname{Diag}\left(s_{1}^{2}, \ldots, s_{n}^{2}\right)$, we may summarize $(16)$ in a more convenient vectorized form as

$$
\frac{\partial \log L}{\partial \boldsymbol{\Lambda}_{n}}=\frac{1}{2} \frac{\mathbf{A}_{n} \mathbf{S} \boldsymbol{\Lambda}_{n}^{-2}+\mathbf{A}_{n}^{-1} \operatorname{Diag}\left(\mathbf{\Upsilon}_{n}^{-1} \overline{\mathbf{Y}}\right)^{2}}{\hat{\nu}_{N}}-\frac{\mathbf{A}_{n}-\mathbf{I}_{n}}{2} \boldsymbol{\Lambda}_{n}^{-1}-\frac{1}{2} \mathbf{A}_{n}^{-1} \operatorname{Diag}\left(\boldsymbol{\Upsilon}_{n}^{-1}\right)
$$

Recall that $s_{i}^{2}=\frac{1}{a_{i}} \sum_{j=1}^{a_{i}}\left(y_{i}^{(j)}-\bar{y}_{j}\right)^{2}$. Therefore, an interpretation of 16 is as extension of the SK estimate $\hat{\sigma}_{i}^{2}$ at $\overline{\mathbf{x}}_{i}$. In contrast with SK, observe that the GP smoothing is precisely what facilitates implementation for small numbers of replicates, even $a_{i}=1$, in which case even though $s_{i}^{2}=0, y_{i}$ still contributes to the local variance estimates via the rest of Eq. (16). Moreover, that smoothing comes nonparametrically via all latent $\delta_{i}$ variance variables. In particular, note that 16 is not constant in $\delta_{i}$; in fact it depends on all of $\boldsymbol{\Delta}_{n}$ via Eq. (14). Remark 4.1. Smoothing may be entertained on other quantities, e.g., $\boldsymbol{\Lambda}_{n} \hat{\nu}_{N}=\mathbf{C}_{(g)} \mathbf{\Upsilon}_{(g)}^{-1} \widehat{\boldsymbol{\Sigma}}_{n}^{2}$ (presuming $a_{i}>1$ ), resulting in smoothed moment-based variance estimates in the style of SK, as advocated by in Kamiński (2015) and Wang and Chen (2016). There may similarly be scope for bypassing a latent GP noise process with the so-called SiNK predictor Lee and Owen, 2015) by taking $\log \boldsymbol{\Lambda}_{n}=\rho(\overline{\mathbf{X}})^{-1} \mathbf{C}_{(g)} \boldsymbol{\Upsilon}_{(g)}^{-1} \boldsymbol{\Delta}_{n}$ with $\rho(\mathbf{x})=\sqrt{\hat{\nu}_{(g)} \mathbf{c}_{(g)}(\mathbf{x})^{\top} \mathbf{\Upsilon}_{(g)}^{-1} \mathbf{c}_{(g)}(\mathbf{x})}$.

\section{An illustration}

The smoothing in Eq. (14) is particularly useful at input locations where the degree of replication is low. For an illustration, consider the motorcycle accident data which is described in more detail in Section 5.1. The SK method would not apply to this data, as inputs are replicated at most six times, and often just once. Since $N=133$ and $n=94$, the gains from the Woodbury trick are minor, yet there is value in properly taking advantage of the few replicates available. The left-hand panels of Figure 1 show the predictive surface(s) in terms of predictive mean (solid red) and its 95\% confidence interval (dashed red) and 95\% prediction interval (dashed green). The right-hand panels show the estimated variance process. The top row corresponds to a homoskedastic fit, the middle row to the non-smoothed 
heteroskedastic case, whereas the bottom row is based on (14). Aesthetically, the bottom
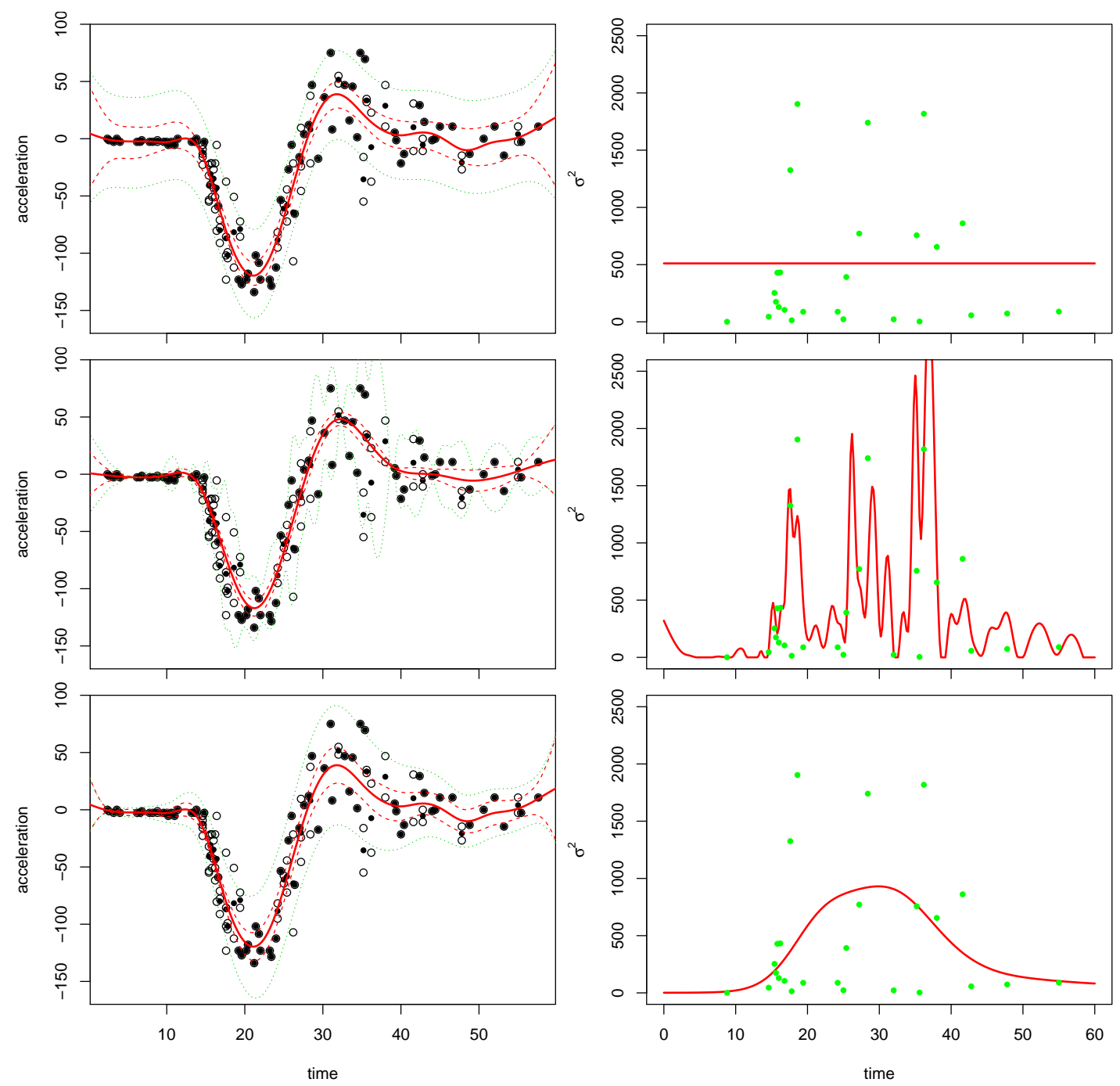

Figure 1: Motorcycle example for constant noise (top), with (bottom) and without (center) smoothing the $\lambda_{i}$ values. Left: mean surface as a solid red line, with $95 \%$ confidence and prediction intervals in red dashed and green dotted lines, respectively. Open circles are actual data points; averaged observations $\bar{y}_{i}$ (for inputs with $a_{i}>1$ ) are filled. Right: means of the variance surface as red lines, empirical variance $\hat{\sigma}_{i}^{2}$ at replicates as green points.

(smoothed variances) look better than the middle (un-smoothed) alternative. A more quantitative comparison, including alternatives from the literature, is provided in Section 5.1 . 


\subsection{Simultaneous optimization of hyperparameters and latents}

Smoothing (14) is key to sensible evolution of the noise process over the input space, but perhaps the most important feature of our framework is how it lends itself to direct and tractable inference via likelihood, without auxiliary calculation or simulation. Computational complexity is linked to the degree of replication $-\mathcal{O}\left(n^{3}\right)$ is much better than $\mathcal{O}\left(N^{3}\right)$ if $N \gg$ $n$-however we remind the reader that no minimal degree of replication is required.

The full set of hyperparameters are determined by the correlation structure(s) of the two GPs, as well as the latent $\boldsymbol{\Delta}_{n}$. We denote by $\boldsymbol{\theta}$ the lengthscales of the mean-field GP, generating the $n \times n$ matrix $\mathbf{C}_{n}$. Similarly, we use $\boldsymbol{\phi}$ for the lengthscales in $\mathbf{C}_{(g)}$, with the degree of smoothing (14) determined by $g$. Inference requires choosing only values of these parameters, $\left\{\boldsymbol{\theta}, \boldsymbol{\phi}, \boldsymbol{\Delta}_{n}, g\right\}$, because conditional on those, the scales $\nu$ and $\nu_{g}$ of both processes have plug-in MLEs: $\hat{\nu}_{N}$ in Eq. (9) and analogously $\hat{\nu}_{(g)}=n^{-1} \boldsymbol{\Delta}_{n}^{\top} \boldsymbol{\Upsilon}_{(g)}^{-1} \boldsymbol{\Delta}_{n}$. Therefore plugging in $\hat{\nu}_{N}$ and $\hat{\nu}_{(g)}$ yields the following concentrated joint $\log$ likelihood $\log \tilde{L}$ :

$$
\begin{aligned}
\log \tilde{L}= & -\frac{N}{2} \log \hat{\nu}_{N}-\frac{1}{2} \sum_{i=1}^{n}\left[\left(a_{i}-1\right) \log \lambda_{i}+\log a_{i}\right]-\frac{1}{2} \log \left|\mathbf{\Upsilon}_{n}\right| \\
& -\frac{n}{2} \log \hat{\nu}_{(g)}-\frac{1}{2} \log \left|\Upsilon_{(g)}\right|+\text { Const }
\end{aligned}
$$

where the top line above is the mean-field component, identical to (8), and the bottom one is the analog for the latent variance process, all up to an additive constant.

Although seemingly close to the penalization used in Quadrianto et al. (2009), a key difference here lies in the use of $g$, analogous to a homogeneous noise on the variance process. Smoothing, via $g$, has an annealing effect on the optimization objective and, as Lemma 4.1 establishes, can have no adverse effect on the final solution. Our own preliminary empirical work (not shown) echoes results of Lazaro-Gredilla and Titsias (2011) who demonstrate the value of smoothing in this context as a guard against overfit. 
Lemma 4.1. The objective $\log \tilde{L}$ in Eq. (18) is maximized when $\boldsymbol{\Delta}_{n}=\log \boldsymbol{\Lambda}_{n}$ and $g=0$.

The proof is left in Appendix B.1, however some discussion may be beneficial. This result says that smoothing (14) is redundant under the GP prior for $\boldsymbol{\Delta}_{n}$ : you'll get a smooth answer anyway, if you find the global maximum. If we initialize $\boldsymbol{\Delta}_{n}$ at a non-smooth setting, say via an empirical estimate of variance derived from residual sums of squares from an initial GP fit, an (initially) non-zero $g$-value will compensate and automatically generate a spatially smooth $\boldsymbol{\Lambda}_{n}$. As the solver progresses we can signal convergence-to a smoothed $\hat{\boldsymbol{\Delta}}_{n}$-if $\hat{g}$ is (numerically) zero. Or, if on a tight computational budget, we can stop the solver early, and rely on $\log \hat{\boldsymbol{\Lambda}}_{n}$ values being smooth, but close to their $\hat{\boldsymbol{\Delta}}_{n}$ counterparts.

Inference for all unknowns may be carried out by a Newton-like scheme since the gradient of (18) is available in closed form. Its components may be derived generically through Eqs. (13 15), i.e., without separating out by hyperparameter, however we detail them below for convenience. For each component $\theta_{k}$ of the mean-field lengthscale $\boldsymbol{\theta}$ defining $\mathbf{C}_{n}$, we have

$$
\frac{\partial \log \tilde{L}}{\partial \theta_{k}}=\frac{1}{2 \hat{\nu}_{N}} \overline{\mathbf{Y}}^{\top} \mathbf{\Upsilon}_{n}^{-1} \frac{\partial \mathbf{C}_{n}}{\partial \theta_{k}} \boldsymbol{\Upsilon}_{n}^{-1} \overline{\mathbf{Y}}-\frac{1}{2} \operatorname{tr}\left(\boldsymbol{\Upsilon}_{n}^{-1} \frac{\partial \mathbf{C}_{n}}{\partial \theta_{k}}\right)
$$

The rest of the components of the gradient depend on the derivative of the smoothed $\boldsymbol{\Lambda}_{n}$, i.e., $\frac{\partial \tilde{L}}{\partial \boldsymbol{\Lambda}_{n}}$ in 17 . For the latent variance parameters $\delta_{i}$ in $\boldsymbol{\Delta}_{n}$, we have

$$
\frac{\partial \log \tilde{L}}{\partial \boldsymbol{\Delta}_{n}}=-\frac{\boldsymbol{\Upsilon}_{(g)}^{-1} \boldsymbol{\Delta}_{n}}{\hat{\nu}_{(g)}}+\mathbf{C}_{(g)} \boldsymbol{\Upsilon}_{(g)}^{-1} \boldsymbol{\Lambda}_{n} \times \frac{\partial \tilde{L}}{\partial \boldsymbol{\Lambda}_{n}}
$$

For each component $\phi_{k}$ of the lengthscale of the noise process, $\boldsymbol{\phi}$, we have

$$
\begin{aligned}
\frac{\partial \log \tilde{L}}{\partial \phi_{k}}= & {\left[\frac{\partial \mathbf{C}_{(g)}}{\partial \phi_{k}}-\mathbf{C}_{(g)} \mathbf{\Upsilon}_{(g)}^{-1} \frac{\partial \mathbf{C}_{(g)}}{\partial \phi_{k}}\right] \boldsymbol{\Upsilon}_{(g)}^{-1} \boldsymbol{\Delta}_{n} \boldsymbol{\Lambda}_{n} \times \frac{\partial \tilde{L}}{\partial \boldsymbol{\Lambda}_{n}} } \\
& +\frac{1}{2 \hat{\nu}_{(g)}} \boldsymbol{\Delta}_{n}^{\top} \mathbf{\Upsilon}_{(g)}^{-1} \frac{\partial \mathbf{C}_{(g)}}{\partial \phi_{k}} \mathbf{\Upsilon}_{(g)}^{-1} \boldsymbol{\Delta}_{n}-\operatorname{tr}\left(\boldsymbol{\Upsilon}_{(g)}^{-1} \frac{\partial \mathbf{C}_{(g)}}{\partial \phi_{k}}\right)
\end{aligned}
$$


And finally, for the noise-smoothing nugget parameter $g$ we have

$$
\begin{aligned}
\frac{\partial \log \tilde{L}}{\partial g}=- & \mathbf{C}_{(g)} \mathbf{\Upsilon}_{(g)}^{-1} \mathbf{A}_{n}^{-1} \mathbf{\Upsilon}_{(g)}^{-1} \boldsymbol{\Delta}_{n} \boldsymbol{\Lambda}_{n} \times \frac{\partial \tilde{L}}{\partial \boldsymbol{\Lambda}_{n}} \\
& +\frac{1}{2 \hat{\nu}_{(g)}} \boldsymbol{\Delta}_{n}^{\top} \mathbf{\Upsilon}_{(g)}^{-1} \mathbf{A}_{n}^{-1} \mathbf{\Upsilon}_{(g)}^{-1} \boldsymbol{\Delta}_{n}-\operatorname{tr}\left(\mathbf{A}_{n}^{-1} \mathbf{\Upsilon}_{(g)}^{-1}\right)
\end{aligned}
$$

\subsection{Implementation details}

Our implementation is in $\mathrm{R}$ (Core Team, 2014), available in the package hetGP Binois and Gramacy (2017), essentially consists of feeding an objective, i.e., a coding of the joint loglikelihood (18), and its gradient 1922 , into the optim library with method="lbfgsb". A demonstration of the library, using the motorcycle data from Sections $4.1 \& 5.1$, is provided in Appendix B.2. The implementation is lean on storage and avoids slow for loops in R. In particular vectors, not matrices, are used to store diagonals like $\boldsymbol{\Delta}_{n}, \mathbf{A}_{n}, \boldsymbol{\Lambda}_{n}$, etc. Traces of non-diagonal matrices are calculated via compiled $\mathrm{C}++$ loops, as are a few other calculations that would otherwise have been cumbersome in R. We have found that a thoughtful initialization of parameters, and in some cases a sensible restriction of their values (i.e., somewhat limiting the parameter space), results in a faster and more reliable convergence on a tight computing budget. E.g., no restarts are required.

Specifically, we find it useful to deploy a priming stage, wherein a single homoskedastic GP (with the Woodbury trick) is fit to the data, in order to set initial values for the latent variables and their GP hyperparameterization. Initial $\delta_{i}$ 's are derived from the logarithm of residual sums of squares calculations on the fitted values obtained from the homoskedastic GP, whose estimated lengthscales determine the initial $\boldsymbol{\theta}$ and $\boldsymbol{\phi}$ values,

$\delta_{i}^{0}=\log \left(1 / a_{i} \sum_{j=1}^{a_{i}}\left(\mu\left(\mathbf{x}_{i}\right)-y_{i}^{(j)}\right)^{2}\right)$. An initial value for $g$ is obtained via a separate, independent homoskedastic GP fit to the resulting collection of $\boldsymbol{\Delta}_{n}$, which may also be utilized to refine the starting $\phi$ values. Our software includes the option of restricting the search 
for lengthscales to values satisfying $\boldsymbol{\phi} \geq \boldsymbol{\theta}$, taken component-wise. This has the effect of forcing the noise process to evolve more slowly in space than the mean-field process does. A thrifty approximate solution that avoids deploying a solver handling constraints involves lower-bounding $\phi$ based on values of $\boldsymbol{\theta}$ obtained from the initial homogeneous fit. We include a further option restricting $\boldsymbol{\phi}=k \boldsymbol{\theta}$ where the scalar $k \geq 1$ is inferred in lieu of $\boldsymbol{\phi}$ directly, which is beneficial in higher dimensions.

Latent variable estimates returned by the optimizer can provide a good indication of the nature of convergence. Any $\delta_{j}$ at the boundary of the $\mathrm{lbfgsb}$ search region either indicates that the bounding box provided is too narrow, the initialization is poor, or that $g$ needs to be larger because the $\boldsymbol{\Lambda}_{n}$ values are being under-smoothed. Although we have iteratively re-engineered our "automatic default" initializations and other settings of the software to mitigate such issues, we find that in fact such adjustments usually have small impact on the overall performance of the method. Numerical experiments backing the proposed initialization scheme are given in Appendix B.3.

After the solver has converged we conclude the procedure with comparison of the unpenalized log-likelihood, i.e., the top part of Eq. (18), to one obtained from an optimized homoskedastic fit. The latter may be higher if the optimization process was unsuccessful, however typically the explanation is that there was not enough data to justify an inputdependent noise structure. In that case we return the superior homoskedastic fit.

Remark 4.2. It is worth noting that working directly with latent variances $\boldsymbol{\Lambda}_{n}$, rather than $\log$ variances $\log \Lambda_{n}$, might be appropriate if it is unreasonable to model the variance evolution multiplicatively. The expressions provided above 1922 simplify somewhat compared to our favored logged version. However, a zero-mean GP prior on the (un-exponentiated) $\boldsymbol{\Lambda}_{n}$ could yield negative predictions, necessitating thresholding. We suggest augmenting with a non-zero constant mean term, i.e., $\hat{\mu}_{(g)}=\mathbf{1}^{\top} \boldsymbol{\Upsilon}_{(g)}^{-1} \boldsymbol{\Delta}_{n}\left(\mathbf{1}^{\top} \boldsymbol{\Upsilon}_{(g)}^{-1} \mathbf{1}\right)^{-1}$ as a potential means of reducing the amount of thresholding required. 


\section{Empirical comparison}

In what follows we consider three examples, starting with a simple expository one from the literature, followed by two challenging "real data" computer model applications from inventory optimization and epidemiology.

\subsection{Benchmark data}

One of the classical examples illustrating heteroskedasticity is the motorcycle accident data. It consists of $N=133$ accelerations with respect to time in simulated crashes, $n=94$ of which are measured at unique time inputs. Hence, this is a somewhat unfavorable example as far as computational advantage goes (via Woodbury), however we note that a method like SK would clearly not apply. Figure 1 provides a visualization of our performance on this data. For the experimental work reported below we use the heteroskedastic fit from the bottom row of that figure, corresponding to smoothed (log) latent variance parameters under full MLE inference for all unknowns, denoted WHGP. See Appendix B.2 for an implementation via mleHetGP in our hetGP library. We also include a homoskedastic GP comparator via our Woodbury computational enhancements, using mleHomGP, abbreviated WGP.

Table 1 summarizes our results alongside ones of other methods in the literature, as extracted from Lazaro-Gredilla and Titsias (2011): another homoskedastic GP without Woodbury (labeled GP), a MAP alternative to the Goldberg et al. (1998) heteroskedastic GP (labeled MAPHGP) from Kersting et al. (2007); Quadrianto et al. (2009) and a variational alternative (VHGP) from Lazaro-Gredilla and Titsias (2011). The experiment involves 300 random $(90 \%, 10 \%)$ partitions into training and testing data, with performance on the testing data averaged over all partitions. Although we generally prefer a Matérn correlation structure, we used a Gaussian kernel for this comparison in order to remain consistent with the other comparators. The metrics used, which are derived from the references above to 


\begin{tabular}{l|c|c|c|c|c} 
& WGP & WHGP & GP $(\star)$ & MAPHGP $(\star)$ & VHGP $(\star)$ \\
\hline NMSE & $0.28 \pm 0.21$ & $0.28 \pm 0.21$ & $0.26 \pm 0.18$ & $0.26 \pm 0.17$ & $0.26 \pm 0.17$ \\
\hline NLPD & $4.59 \pm 0.25$ & $4.26 \pm 0.31$ & $4.59 \pm 0.22$ & $4.32 \pm 0.60$ & $4.32 \pm 0.30$
\end{tabular}

Table 1: Average Normalized MSE and Negative Log-Probability Density (NLPD) \pm 1 sd on the motorcycle data over 300 runs for our methods (WGP and WHGP) compared to those (*) reported in Lazaro-Gredilla and Titsias (2011). Lower numbers are better.

avoid duplicating the efforts in those studies, are Normalized Mean Squared Error (NMSE) and Negative Log-Probability Density (NLPD), the latter being, up to a constant, the negative of the proper scoring rule we prefer in our next example. For details see Lazaro-Gredilla and Titsias (2011). The take-away message from the table is that our proposed methods are comparable, in terms of accuracy, to these comparators. We report that our WHGP method took less than one second to perform 100 optim iterations.

\subsection{Assemble to order}

Here we consider the so-called "assemble-to-order" (ATO) problem first introduced by Hong and Nelson (2006). At its heart it is an optimization (or reinforcement learning) problem, however here we simply treat it as a response surface to be learned. Although the signalto-noise ratio is relatively high, ATO simulations are known to be heteroskedastic, e.g., as illustrated by the documentation for the MATLAB library we utilized for the simulations (Xie et al., 2012). The problem centers around inventory management for a company that manufactures $m$ products. Products are built from base parts, called items, some of which are "key" in that the product cannot be built without them. If a request comes in for a product which is missing one or more key items, a replenishment order is executed, and is filled after a random period. Holding items in inventory is expensive, so there is a balance to be struck between the cost of maintaining inventory, and revenue which can only be realized if the inventory is sufficient to fulfill demand for products.

The inventory costs, product revenue, makeup of products (their items), product demand 
stream and distribution of the random replenishment time of items, together comprise the problem definition. Here we use the canonical specification of Hong and Nelson (2006) involving five products built from eight items. The input space is a target stock vector $b \in\{0,1, \ldots, 20\}^{8}$ for the item inventories, and the ATO simulator provides a Monte Carlo estimate of expected daily profit under that regime.

For the experiment reported on below we evaluated ATO on a Latin Hypercube sample of size 2000 in the discrete 8-dimensional space, sampling ten replicates at each site. We then embarked on fifty Monte Carlo repetitions of an out-of-sample predictive comparison obtained by partitioning the data into training and testing sets in the following way. We randomly chose half $(n=1000)$ of the sites as training sites, and at each site collected $a_{i} \sim$ Unif $\{1, \ldots, 10\}$ training responses among the ten replicates available. In this way, the average size of the training data was $N=5000$. The ten replicates at the remaining $n=1000$ testing sites comprise our out-of-sample testing set (for 10000 total). We further retain the random number of unchosen (i.e., also out-of-sample) training replicates (of which there are 5000 on average) as further testing locations.

The left panel of Figure 2 summarizes the distribution of scores combining predictions of the mean and variance together via the proper scoring rule provided by Eq. (27) of Gneiting and Raftery (2007). The plot is divided into four segments, and in the first three the same six comparators are entertained. The first segment tallies scores on the testing design locations, the third segment on the (out-of-sample) training locations, and the second segment combines those two sets. The final segment shows the purely in-sample performance of a $\left(\bar{y}_{i}, s_{i}^{2}\right)$ estimator ("avg") for benchmarking purposes. The six comparators, from left to right, are "Hom $\bar{Y}$ " (a homoskedastic GP trained on $n=1000$ pairs $(\bar{Y}, \bar{X})$, i.e., otherwise discarding the replicates), "Hom $Y^{(1)}$ " (a homoskedastic GP trained on $n=1000$ runs comprised of only one (namely first) replicate in the training data at each site), "WHom $Y$ " (a homoskedastic GP on the full training runs using the Woodbury trick), "Hom Y" 

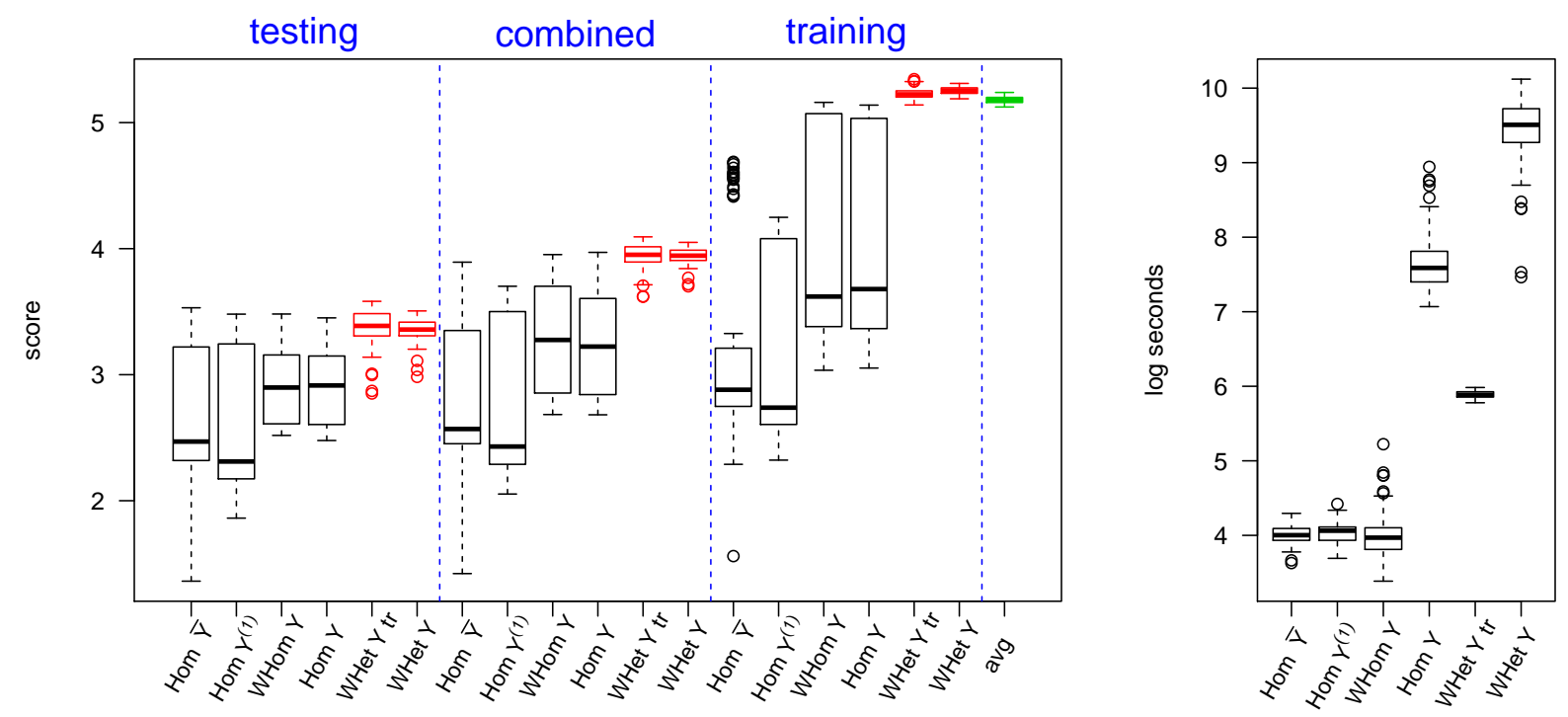

Figure 2: The right panel shows scores (higher is better) on ATO data comparing homoskedastic partial and full-data comparators, and heteroskedastic comparators (in red). Sets are partitioned into pure out-of-sample "testing", out-of sample "training" at the inputdesign locations, and "combined". The green right-most boxplot corresponds to the empirical mean and variance comparator at the "training" sites. The right panel shows computing times in log seconds.

(a homoskedastic GP on the full training runs without the Woodbury trick), "WHet $Y$ tr" (a truncated heteroskedastic GP on the full training runs, using the Woodbury trick but stopping after 100 optim iterations), and finally "WHet $Y$ ", which is the same without limiting optim. To ease viewing, the heteroskedastic comparators are colored in red.

Although there is some nuance in the relative comparison of the boxplots, the takehome message is that the heteroskedastic comparators are the clear winners. The fact that on the (out-of-sample) training runs the heteroskedastic comparator is better than "avg" indicates that smoothed variances are as crucial for good performance at design sites as it is elsewhere (where smoothing is essential). The wall-clock times of the relative comparators are summarized in the right panel of the figure. Observe that "WHom $Y$ " is much faster than "Hom $Y$ " with a speed-up of about $40 \times$. This illustrates the value of the Woodbury identities; recall that here $n \approx N / 5$. Also, comparing "WHet $Y \operatorname{tr}$ " and "WHet $Y$ ", we see 
that there is little benefit from running optim to convergence, which often consumes the vast bulk of the computing time (a difference of over $50 \times$ in the figure), yet yields minimal improvement in the score for these comparators.

\subsection{Epidemics management}

Our last example concerns an optimization/learning problem that arises in the context of designing epidemics countermeasures (Ludkovski and Niemi, 2010; Merl et al., 2009). Infectious disease outbreaks, such as influenza, dengue fever and measles, spread stochastically through contact between infected and susceptible individuals. A popular method to capture outbreak uncertainty is based on constructing a stochastic compartmental model that partitions the population into several epidemiological classes and prescribes the transition rates among these compartments. One of the simplest versions is the stochastic SIR model that is based on Susceptible, Infected and Recovered counts, with individuals undertaking $S \rightarrow I$ and $I \rightarrow R$ moves. In a continuous-time formulation, the state $\mathbf{x}(t):=\left(S_{t}, I_{t}, R_{t}\right), t \in \mathbb{R}_{+}$ is a Markov chain taking values on the simplex $\mathcal{X}_{M}=\left\{(s, i, r) \in \mathbb{Z}_{+}^{3}, s+i+r \leq M\right\}$ where $M$ is the total population size, here fixed for convenience. The SIR system has two possible transition channels, with corresponding rates (Hu and Ludkovski, 2017)

$$
\left\{\begin{array}{ll}
\text { Infection: } S+I \rightarrow 2 I & \text { with rate } \beta S_{t} I_{t} / M ; \\
\text { Recovery: } I \rightarrow R & \text { with rate } \gamma I_{t} .
\end{array}\right\}
$$

Policy makers aim to dynamically enact countermeasures in order to mitigate epidemic costs; a simple proxy for the latter is the total number of individuals who are newly infected $f(\mathbf{x}):=\mathbb{E}\left[S_{0}-\lim _{T \rightarrow \infty} S_{T} \mid\left(S_{0}, I_{0}, R_{0}\right)=\mathbf{x}\right]=\gamma \mathbb{E}\left[\int_{0}^{\infty} I_{t} d t \mid \mathbf{x}\right]$. Due to the nonlinear transition rates, there is no closed-form expression for the above expectation, however it can be readily estimated via Monte Carlo by generating outbreak trajectories and averaging. 
The final optimization step, which we do not consider here, performs a cost-benefit analysis by comparing expected costs under different policy regimes (e.g., "do-nothing", "public information campaign", "vaccination campaign").

The form of (23) induces a strong heteroskedasticity in the simulations. For low infected counts $I \leq 10$, the outbreak is likely to die out quickly on its own, leading to low expected infecteds and low simulation variance. For intermediate values of $I_{0}$, there is a lot of variability across scenarios, yielding high conditional variance $r(\mathbf{x})$. However, if one starts with a lot of initial infecteds, the dynamics become essentially deterministic due to the underlying law of large numbers, leading to large costs but low simulation noise. The demarcation of these regions is driven by initial susceptible count and the transition rates $\beta, \gamma$ in (23).

As proof of concept, we consider learning $f$ and $r$ for the case $\beta=\gamma=0.5, M=2000$. The input space is restricted to $\{(S, I): S \in\{1200, \ldots, 1800\}, I \in\{0, \ldots, 200\}\}$. Outside those ranges the outbreak behavior is trivial. Inside the region, simulation noise ranges from $r(\mathbf{x})=0$ at $I_{0}=0$ to as high as $r(\mathbf{x})=86^{2}$. Figure 6 in $\mathrm{Hu}$ and Ludkovski (2017) shows a variance surface generated via SK using $a_{i} \equiv 100$ replicates at each input and an adaptive design. Here we propose a more challenging setup where the number of replicates is not fixed and includes small counts. To this end, we generate a large training set with slightly more than two thousand locations on a regular grid, with a thousand replications. Then we compare the results given by SK and our method based on a random subset of $n=1000$ $\mathbf{x}_{i}$ 's with a varying number of replicates. Specifically, 500 design sites have only 5 replicates (250 with 10, 150 with 50 and 100 with 100, respectively). To account for variance known to be zero at $I=0$, all design points on this domain boundary are artificially replicated 100 times, so $N=\sum_{i} a_{i}=24205$ on this specific instance.

The counterpart of Figure 6 in $\mathrm{Hu}$ and Ludkovski (2017) is Figure 3 here. Our proposed method recovers the key features of the variance surface, with a significantly lower number of replicates. As a comparison (see rightmost panel), an interpolating model directly fit 


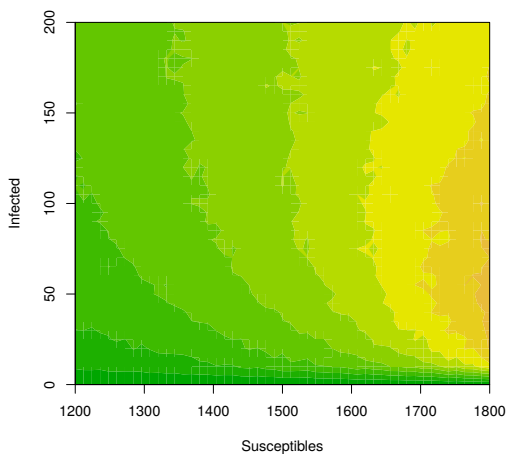

(a) Reference

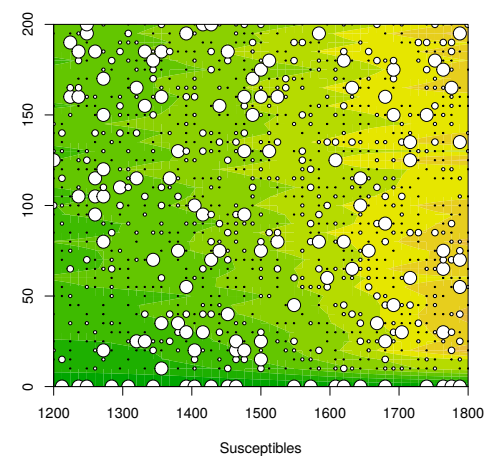

(b) Joint estimation

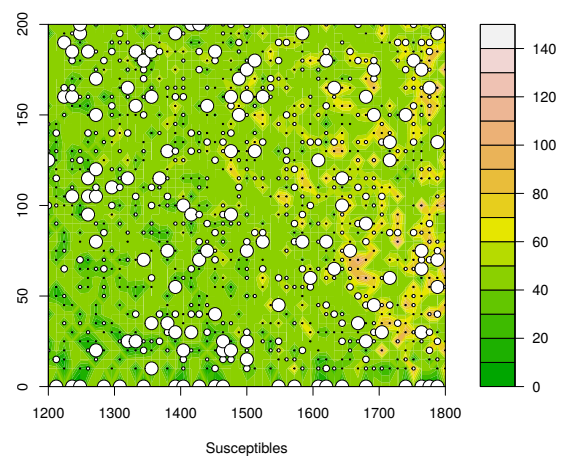

(c) Empirical (interpolated)

Figure 3: Estimated standard deviation surfaces for the epidemics example with several methods. Left: reference empirical standard deviation on all two thousand locations and all thousand replicates. Center and right: results based on one thousand locations with varying number of replications, depicted by points of increasing size (i.e., for 5, 10, 50 and 100 replicates). Center: results given from the joint estimation procedure. Right: interpolation of the empirical standard deviation at given $\mathbf{x}_{i}{ }^{\prime}$ s.

onto the empirical variances, as suggested in Ankenman et al. (2010) is much less accurate. It is then not surprising that SK is impacted by the inaccuracies of the empirical mean and variance estimates. In particular, we show in the left panel of Figure 4 that the mean prediction for SK is off even at inputs with many replicates $\left(a_{i}=50\right.$ or $\left.a_{i}=100\right)$, while our method benefits from the smoothing of latent variables. In addition, we can perform the same analysis on the predicted variance, showing again in the right panel of Figure 4 that our estimates outperform the use of the empirical $\hat{\sigma}_{i}$ 's, even for the largest number of replicates. Observe that the empirical estimates are notably high-biased when $a_{i}<50$.

\section{Discussion}

We have enhanced heteroskedastic Gaussian process modeling by, effectively, combining the strengths of canonical approaches in two disparate literatures, namely machine learning (via Goldberg et al.) and industrial design (via Ankenman et al.). We introduced two novel aspects, the Woodbury trick for computational shortcuts (without approximation), and a 

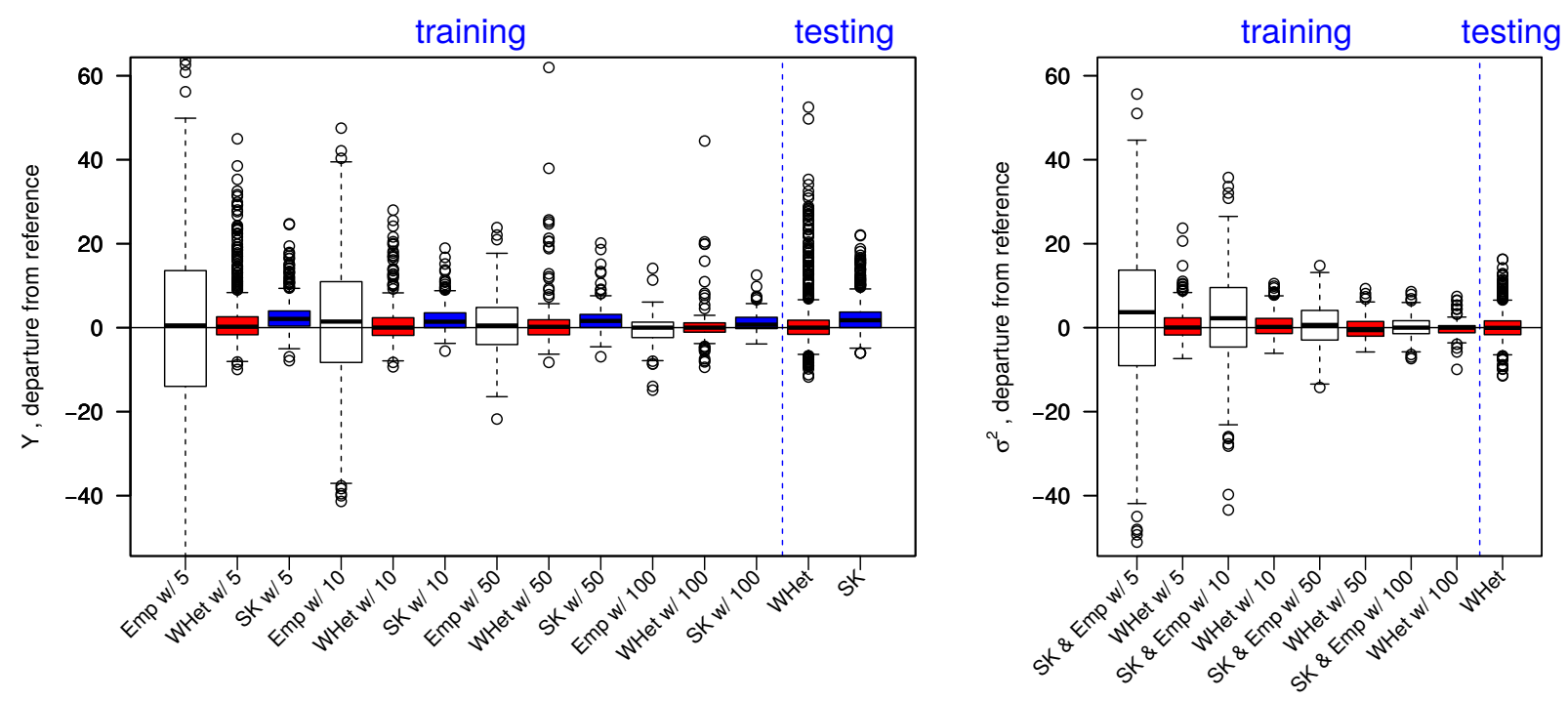

Figure 4: Boxplot of difference between reference mean (left panel) and reference standard deviation (right panel) obtained on all data with, on a subset of locations and replicates, the empirical mean (Emp, white), the results given by Stochastic Kriging (SK, blue) and those of our method (WHet, red). Results are separated by the number of replicates in the training set (5 to 100), as well as comparison on the testing set (extreme right).

smoothed latent process in lieu of expensive EM and MCMC simulation-based alternatives to inference. The result is an inferential framework that upgrades modeling fidelity -inputdependent noise - while retaining a computational complexity on par with the best implementations of constant noise GP methods. Appendix C explores how our approach copes well with other types of non-stationarity, despite the model mismatch.

In our survey of the literature we discovered a connection between the Woodbury trick and an approximate method of circumventing big data (homoskedastic) GP calculations via pseudo inputs (Snelson and Ghahramani, 2005) and the so-called predictive process Banerjee et al. (2008). Those methods, rather than leveraging replicates, approximates a big data spatial field with a smaller latent process: the inputs, outputs, and parameters for which are jointly inferred in a unified optimization (pseudo-inputs) or Bayesian (predictive process) framework. Indeed the spirit of pseudo-input version is similar to ours, being almost identical when restricting the latent process to the coordinates of the unique- $n$ inputs $\overline{\mathbf{X}}$ in 
the homoskedastic modeling setup. Snelson and Gharamani (2006) and later Kersting et al. (2007) proposed heteroskedastic pseudo-inputs variants, with raw optimization of the latents rather than via the smoothing process that we propose. An advantage of free estimation of the pseudo inputs, i.e., rather than fixing them to $\overline{\mathbf{X}}$ as we do, is that additional computational savings can be realized in situations where replication is infeasible or undesired, albeit by approximation and assuming that the pseudo-inputs can be selected efficiently. We see tremendous potential for further hybridization of our approach with the wider literature on approximate (heteroskedastic) modeling of GP in big data contexts.

\section{Acknowledgments}

All three authors are grateful for support from National Science Foundation grants DMS1521702 and DMS-1521743. Many thanks to Peter Frazier for suggesting the ATO problem.

\section{A Supporting replication}

The subsections below provide some of the details that go into lemmas involving the Woodbury trick for efficient inference and prediction with designs under replication, followed by an empirical illustration of the potential computational savings using our library routines.

\section{A.1 Derivations supporting lemmas in Section 3}

Due to the structure of replicates, it follows that $\mathbf{U}^{\top} \mathbf{U}=\mathbf{A}_{n}, \mathbf{A}_{n} \mathbf{k}_{n}(\mathbf{x})=\mathbf{U}^{\top} \mathbf{k}(\mathbf{x}), \mathbf{U k}_{n}(\mathbf{x})=$ $\mathbf{k}\left(\mathbf{x}\right.$ ). Moreover, $\mathbf{A}_{n} \overline{\mathbf{Y}}=\mathbf{U}^{\top} \mathbf{Y}, \mathbf{U}^{\top} \boldsymbol{\Sigma}_{N}=\boldsymbol{\Sigma}_{n} \mathbf{U}^{\top}$, $\mathbf{U} \boldsymbol{\Sigma}_{n}=\boldsymbol{\Sigma}_{N} \mathbf{U}, \mathbf{A}_{n} \boldsymbol{\Sigma}_{n}=\mathbf{U}^{\top} \boldsymbol{\Sigma}_{N} \mathbf{U}$ (formu- 
las with $\boldsymbol{\Sigma}_{N}$ work with $\boldsymbol{\Sigma}_{N}^{-1}$ as well). Woodbury's identity gives (4):

$$
\begin{aligned}
\mathbf{k}_{n}(\mathbf{x})^{\top} & \left(\mathbf{K}_{n}+\mathbf{A}_{n}^{-1} \boldsymbol{\Sigma}_{n}\right)^{-1} \overline{\mathbf{Y}}=\mathbf{k}_{n}(\mathbf{x})^{\top} \boldsymbol{\Sigma}_{n}^{-1} \mathbf{A}_{n} \overline{\mathbf{Y}}-\mathbf{k}_{n}(\mathbf{x})^{\top} \mathbf{A}_{n} \boldsymbol{\Sigma}_{n}^{-1}\left(\mathbf{K}_{n}^{-1}+\mathbf{A}_{n} \boldsymbol{\Sigma}_{n}^{-1}\right)^{-1} \boldsymbol{\Sigma}_{n}^{-1} \mathbf{A}_{n} \overline{\mathbf{Y}} \\
& =\mathbf{k}_{n}(\mathbf{x})^{\top} \boldsymbol{\Sigma}_{n}^{-1} \mathbf{U}^{\top} \mathbf{Y}-\mathbf{k}(\mathbf{x})^{\top} \mathbf{U} \boldsymbol{\Sigma}_{n}^{-1}\left(\mathbf{K}_{n}^{-1}+\mathbf{A}_{n} \boldsymbol{\Sigma}_{n}^{-1}\right)^{-1} \boldsymbol{\Sigma}_{n}^{-1} \mathbf{U}^{\top} \mathbf{Y} \\
& =\mathbf{k}_{n}(\mathbf{x})^{\top} \mathbf{U}^{\top} \boldsymbol{\Sigma}_{N}^{-1} \mathbf{Y}-\mathbf{k}(\mathbf{x})^{\top} \boldsymbol{\Sigma}_{N}^{-1} \mathbf{U}\left(\mathbf{K}_{n}^{-1}+\mathbf{A}_{n} \boldsymbol{\Sigma}_{n}^{-1}\right)^{-1} \mathbf{U}^{\top} \boldsymbol{\Sigma}_{N}^{-1} \mathbf{Y} \\
& =\mathbf{k}(\mathbf{x})^{\top} \boldsymbol{\Sigma}_{N}^{-1} \mathbf{Y}-\mathbf{k}(\mathbf{x})^{\top} \boldsymbol{\Sigma}_{N}^{-1} \mathbf{U}\left(\mathbf{K}_{n}^{-1}+\mathbf{U}^{\top} \boldsymbol{\Sigma}_{N}^{-1} \mathbf{U}\right)^{-1} \mathbf{U}^{\top} \boldsymbol{\Sigma}_{N}^{-1} \mathbf{Y}=\mathbf{k}(\mathbf{x})^{\top}\left(\mathbf{K}+\boldsymbol{\Sigma}_{N}\right)^{-1} \mathbf{Y}
\end{aligned}
$$

The calculations are the same when replacing $\overline{\mathbf{Y}}$ by $\mathbf{k}_{n}(\mathbf{x})$ on the right. On the other hand, having $\mathbf{Y}$ on both sides yields:

$$
\begin{aligned}
\mathbf{Y}^{\top}\left(\mathbf{K}_{N}+\boldsymbol{\Sigma}_{N}\right)^{-1} \mathbf{Y} & =\mathbf{Y}^{\top} \boldsymbol{\Sigma}_{N}^{-1} \mathbf{Y}-\mathbf{Y}^{\top} \boldsymbol{\Sigma}_{N}^{-1} \mathbf{U}\left(\mathbf{K}_{n}^{-1}+\mathbf{U}^{\top} \boldsymbol{\Sigma}_{N}^{-1} \mathbf{U}\right)^{-1} \mathbf{U}^{\top} \boldsymbol{\Sigma}_{N}^{-1} \mathbf{Y} \\
& =\mathbf{Y}^{\top} \boldsymbol{\Sigma}_{N}^{-1} \mathbf{Y}-\mathbf{Y}^{\top} \mathbf{U} \boldsymbol{\Sigma}_{n}^{-1}\left(\mathbf{K}_{n}^{-1}+\mathbf{A}_{n} \boldsymbol{\Sigma}_{n}^{-1}\right)^{-1} \boldsymbol{\Sigma}_{n}^{-1} \mathbf{U}^{\top} \mathbf{Y} \\
& =\mathbf{Y}^{\top} \boldsymbol{\Sigma}_{N}^{-1} \mathbf{Y}-\overline{\mathbf{Y}}^{\top} \mathbf{A}_{n} \boldsymbol{\Sigma}_{n}^{-1}\left(\mathbf{K}_{n}^{-1}+\mathbf{A}_{n} \boldsymbol{\Sigma}_{n}^{-1}\right)^{-1} \boldsymbol{\Sigma}_{n}^{-1} \mathbf{A}_{n} \overline{\mathbf{Y}} \\
& =\mathbf{Y}^{\top} \boldsymbol{\Sigma}_{N}^{-1} \mathbf{Y}-\overline{\mathbf{Y}}^{\top} \mathbf{A}_{n} \boldsymbol{\Sigma}_{n}^{-1} \overline{\mathbf{Y}}+\overline{\mathbf{Y}}^{\top}\left(\mathbf{K}_{n}+\mathbf{A}_{n}^{-1} \boldsymbol{\Sigma}_{n}\right)^{-1} \overline{\mathbf{Y}}
\end{aligned}
$$

The determinant is similar:

$$
\begin{aligned}
\log \left|\mathbf{K}+\boldsymbol{\Sigma}_{N}\right| & =\log \left|\mathbf{K}_{n}^{-1}+\mathbf{U}^{\top} \boldsymbol{\Sigma}_{N}^{-1} \mathbf{U}\right|+\log \left|\mathbf{K}_{n}\right|+\log \left|\boldsymbol{\Sigma}_{N}\right| \\
& =\log \left|\mathbf{K}_{n}\right|+\log \left|\mathbf{K}_{n}^{-1}+\mathbf{A}_{n} \boldsymbol{\Sigma}_{n}^{-1}\right|+\sum_{i=1}^{n} a_{i} \log \Sigma_{i} \\
& =\log \left|\mathbf{K}_{n}+\mathbf{A}_{n}^{-1} \boldsymbol{\Sigma}_{n}\right|+\sum_{i=1}^{n}\left[\left(a_{i}-1\right) \log \Sigma_{i}+\log a_{i}\right] \\
& =\log \left|\mathbf{K}_{n}+\mathbf{A}_{n}^{-1} \boldsymbol{\Sigma}_{n}\right|+\log \left|\boldsymbol{\Sigma}_{N}\right|-\log \left|\mathbf{A}_{n}^{-1} \boldsymbol{\Sigma}_{n}\right|
\end{aligned}
$$




\section{A.2 Empirical demonstration of Woodbury speedups}

The $\mathrm{R}$ code below sets up a design in 2- $\mathrm{d}$ and evaluates the response as $y(\mathbf{x})=x_{1} \exp \left\{-x_{1}^{2}-\right.$ $x_{2}^{2}$ ) (Gramacy, 2007), observed with $\mathcal{N}\left(0,0.01^{2}\right)$ noise. Our example in Appendix Cp provides a visual [in Figure 7] of this response in a slightly different context. The design has $n=100$ unique space-filling locations, where their degree of replication is determined uniformly at random in $\{1, \ldots, 50\}$ so that, on average, there are $N=2500$ total elements in the design.

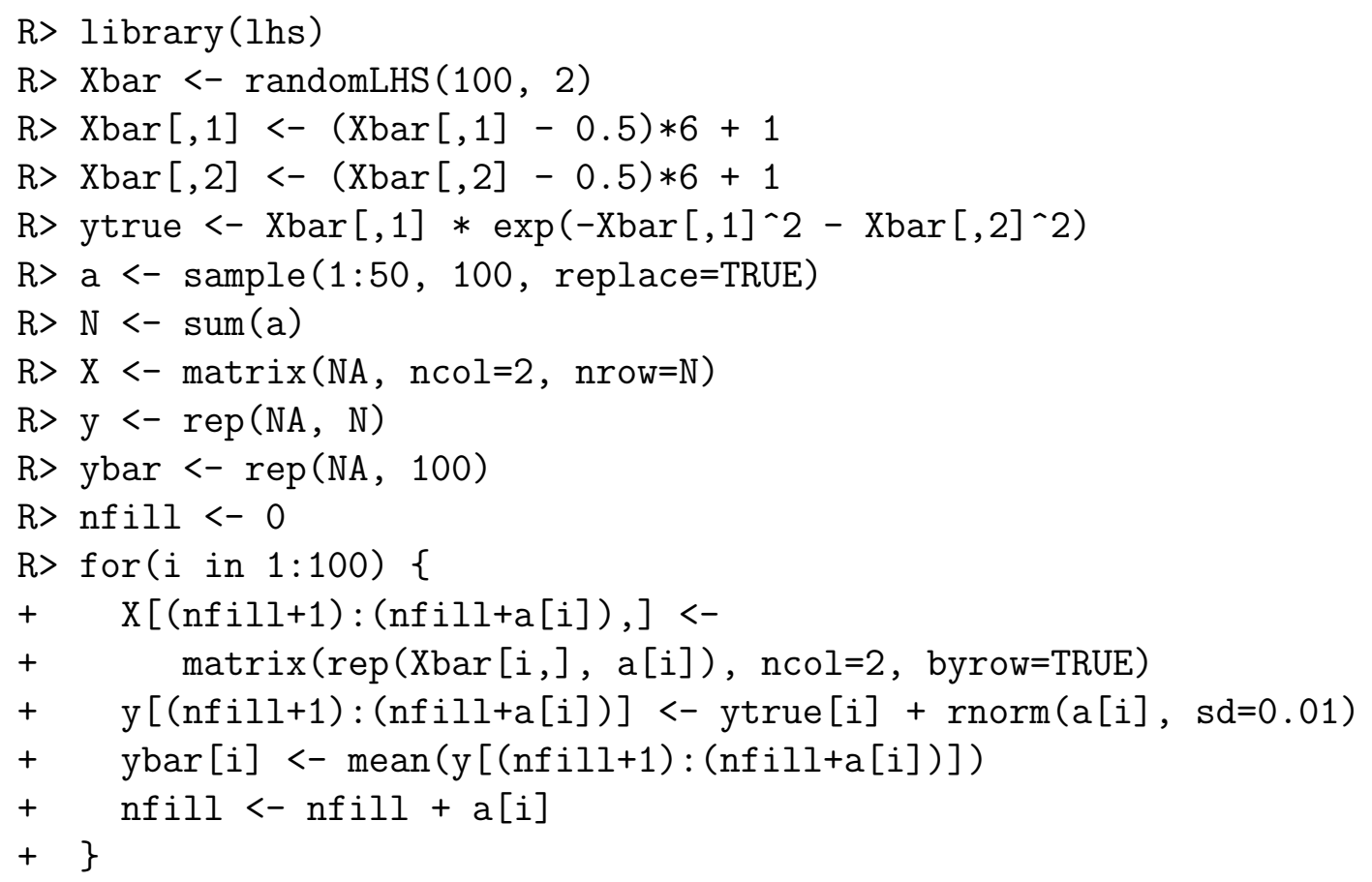

Below, we use the homoskedastic modeling function mleHomGP from our hetGP package in two ways. First, we use it as intended, with the Woodbury trick, saving the the wall time.

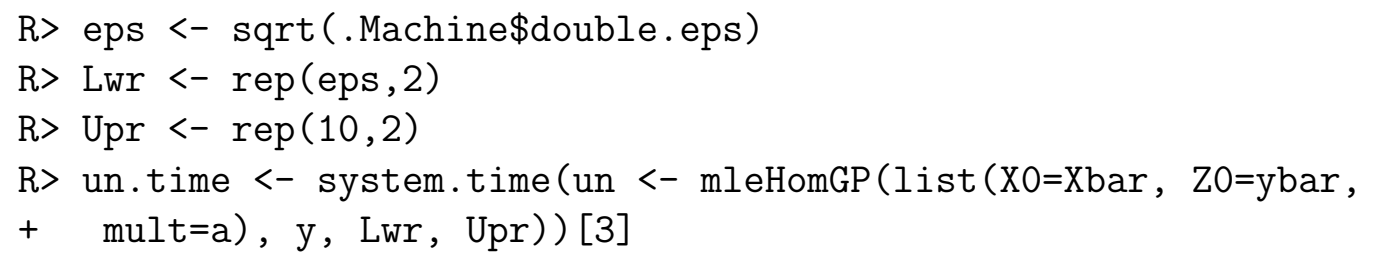

Then, we use exactly the same function, but with arguments that cause the Woodbury trick to be bypassed - essentially telling the implementation that there are no replicates. 
$\mathrm{R}>\mathrm{fN}$. time <- system.time $(\mathrm{fN}<-$ mleHomGP(list $(\mathrm{X} 0=\mathrm{X}, \mathrm{ZO}=\mathrm{y}$,

$+\operatorname{mult}=\operatorname{rep}(1, \mathrm{~N}))$, y, Lwr, Upr)) [3]

First, lets compare times.

$R>c(f N=f N \cdot$ time, un=un.time $)$

\#\# fN.elapsed un.elapsed

$\begin{array}{lll}\text { \#\# } \quad 432.281 & 0.093\end{array}$

Observe that the Woodbury trick version is more than 4000 times faster. This experiment was run an eight-core Intel i7 with 32 GB of RAM with R's default linear algebra libraries (i.e., not the threaded MKL library used for ATO experiment in Section 5.2).

Just to check that both methods are performing the same inference, the code chunk below prints the pairs of estimated lengthscales $\hat{\theta}$ to the screen.

$\mathrm{R}>\operatorname{rbind}(\mathrm{f}=\mathrm{fN} \$$ theta, un=un $\$$ theta $)$

\#\# [, 1] [, 2]

\#\# fN $1.099367 \quad 1.789508$

\#\# un 1.0993081 .789700

Above we specified the the $\mathrm{X}$ input as a list with components X0, Z0 and mult to be explicit about the mapping between unique- $n$ and full- $N$ representations. However, this is not the default (or intended) mechanism for providing the design, which is to simply provide a matrix $\mathrm{X}$. In that case, the implementation first internally calls the find_reps function in order to locate replicates in the design, and build the appropriate list structure for further calculation.

\section{B Supporting heteroskedastic elements}

Here we provide derivations supporting our claim that smoothed and optimal solutions (for latent variables) coincide at the maximum likelihood setting. We then provide a het- 
eroskedastic example using the hetGP library. Finally, we provide an empirical demonstration illustrating the consistency of hetGP optimization of the latent variance variables.

\section{B.1 Proof of Lemma 4.1}

Proof. Denote $\left(\boldsymbol{\theta}^{*}, \boldsymbol{\Delta}_{n}^{*}, \boldsymbol{\phi}^{*}, g^{*}\right)$ as the maximizer of Eq. 18). For simplicity, we work here with variances and not log variances. By definition, $\boldsymbol{\Lambda}_{n}^{*}=\mathbf{C}_{(g)} \mathbf{\Upsilon}_{(g)}^{-1} \boldsymbol{\Delta}_{n}^{*}$ if and only if $\boldsymbol{\Upsilon}_{(g)} \mathbf{C}_{(g)}^{-1} \boldsymbol{\Lambda}_{n}^{*}=\boldsymbol{\Delta}_{n}^{*}$. Notice that the top part of $\sqrt{18}$ is invariant as long as $\boldsymbol{\Lambda}_{n}=\boldsymbol{\Lambda}_{n}^{*}$. We thus concentrate on the remaining terms: $\log \left(\nu_{(g)}\right)$ and $\log \left|\mathbf{\Upsilon}_{(g)}\right|$. First, the derivative of $\log \left|\mathbf{\Upsilon}_{(g)}\right|$ with respect to $g$ is $\operatorname{tr}\left(\mathbf{A}_{n}^{-1} \mathbf{\Upsilon}_{(g)}^{-1}\right)$, which is positive (trace of a positive definite matrix), hence $\log \left|\Upsilon_{(g)}\right|$ increases with $g$; it also does not depend on $\boldsymbol{\Delta}_{n}$. As for $\log \left(\nu_{(g)}\right)$, $n \nu_{(g)}=\boldsymbol{\Delta}_{n}^{* \top} \boldsymbol{\Upsilon}_{(g)}^{-1} \boldsymbol{\Delta}_{n}^{*} \geq \boldsymbol{\Lambda}_{n}^{* \top} \mathbf{C}_{(g)}^{-1} \boldsymbol{\Lambda}_{n}^{*}$ since

$$
\boldsymbol{\Delta}_{n}^{* \top} \boldsymbol{\Upsilon}_{(g)}^{-1} \boldsymbol{\Delta}_{n}^{*}=\boldsymbol{\Lambda}_{n}^{* \top} \mathbf{C}_{(g)}^{-1} \boldsymbol{\Upsilon}_{(g)} \boldsymbol{\Upsilon}_{(g)}^{-1} \boldsymbol{\Upsilon}_{(g)} \mathbf{C}_{(g)}^{-1} \boldsymbol{\Lambda}_{n}^{*}=\boldsymbol{\Lambda}_{n}^{* \top} \mathbf{C}_{(g)}^{-1} \boldsymbol{\Lambda}_{n}^{*}+g \boldsymbol{\Lambda}_{n}^{* \top} \mathbf{C}_{(g)}^{-1} \mathbf{A}_{n}^{-1} \mathbf{C}_{(g)}^{-1} \boldsymbol{\Lambda}_{n}^{*}
$$

and $\mathbf{C}_{(g)}^{-1} \mathbf{A}_{n}^{-1} \mathbf{C}_{(g)}^{-1}$ is positive definite. Hence $g^{*}=0$ and $\boldsymbol{\Lambda}_{n}^{*}=\boldsymbol{\Delta}_{n}^{*}$.

\section{B.2 Illustration in hetGP}

The $\mathrm{R}$ code below reproduces the key aspects our application of hetGP on the motorcycle data (available, e.g., in the package MASS Venables and Ripley (2002)), in particular the bottom row of Figure 1.

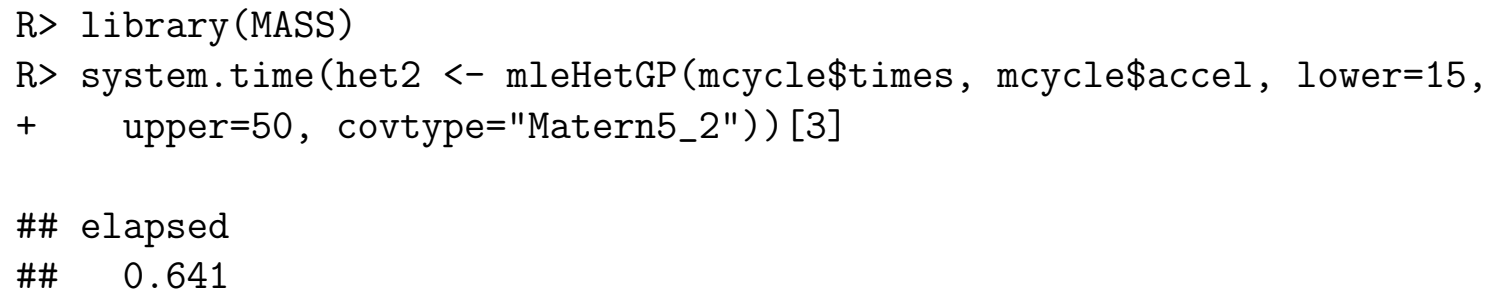

So it takes about one second to optimize over the unknown parameters, including the latent 
variances, when linked to R's default linear algebra libraries. Observe that raw inputs (times) and responses (accel) are provided, trigging a call to the package's internal find_reps function to navigate the small amount replication in the design via the Woodbury trick.

The plotting code below generates a pair of plots similar to those in the final row of Figure 1. which we do not duplicate here. Code for other examples, including the more expensive Monte Carlo experiments for our ATO and SIR examples, is available upon request.

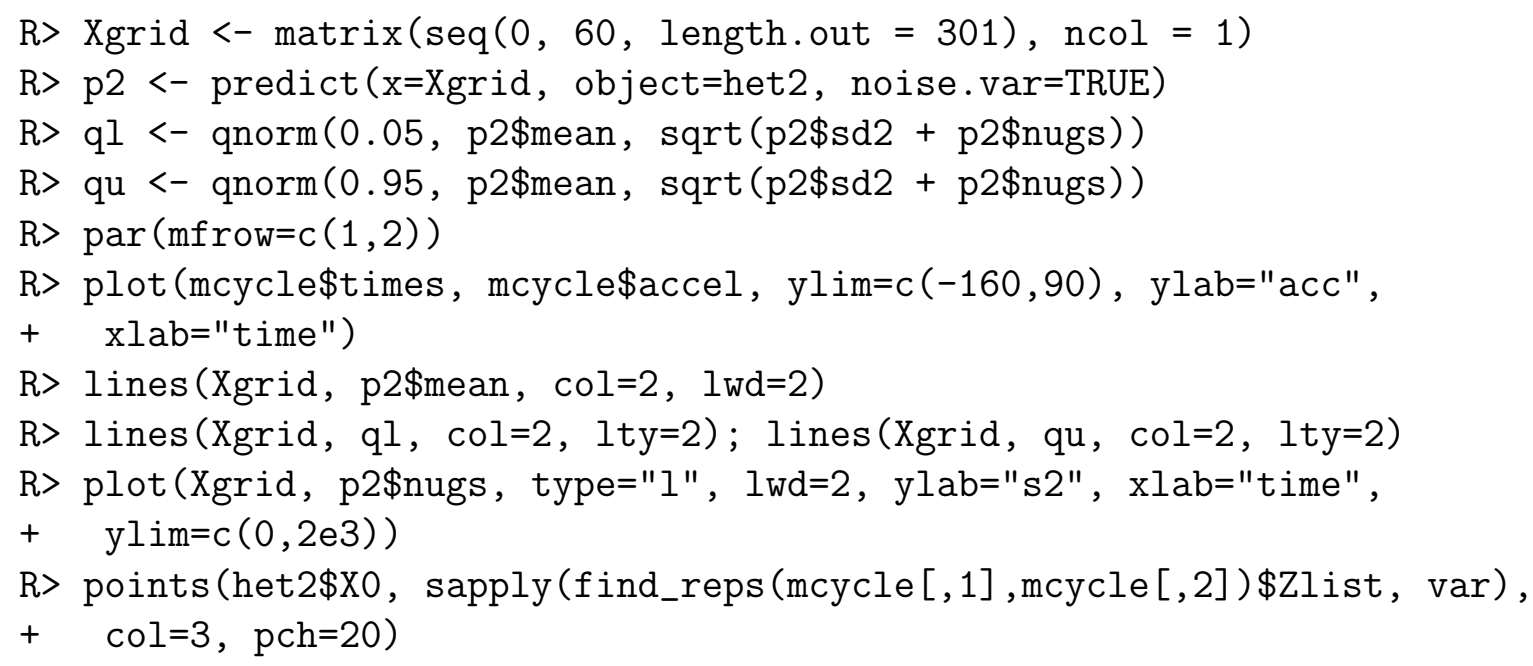

\section{B.3 Robustness in numerical optimization}

In Section 4.3 we advocate initializing gradient-based optimization of the latent noise variables using residuals obtained from homogeneous fit, and this is the default in hetGP. To illustrate that this is a good starting point, difficulties in MLE estimation for GP hyperparameterization notwithstanding (Erickson et al., 2017), we provide here two demonstrations that this procedure is efficient. The first example reuses the motorcycle data while the second is the 2d Branin function (Dixon and Szego, 1978) redefined on $[0,1]^{2}$ with i.i.d Gaussian additive noise with variance: $\left.\left.2+2 \sin \left(\pi x_{1}\right) \cos \left(3 \pi x_{2}\right)+5\left(x_{1}^{2}+x_{2}^{2}\right)\right)\right)$. Unique $n=100$ design points have been sampled uniformly, along with uniformly allocated replicates until $N=1000$. The experiments compare the log-likelihood and other estimates obtained via 
L-BFGS-B optimization under our default initialization, and collection of random ones.

\section{Motorcycle}
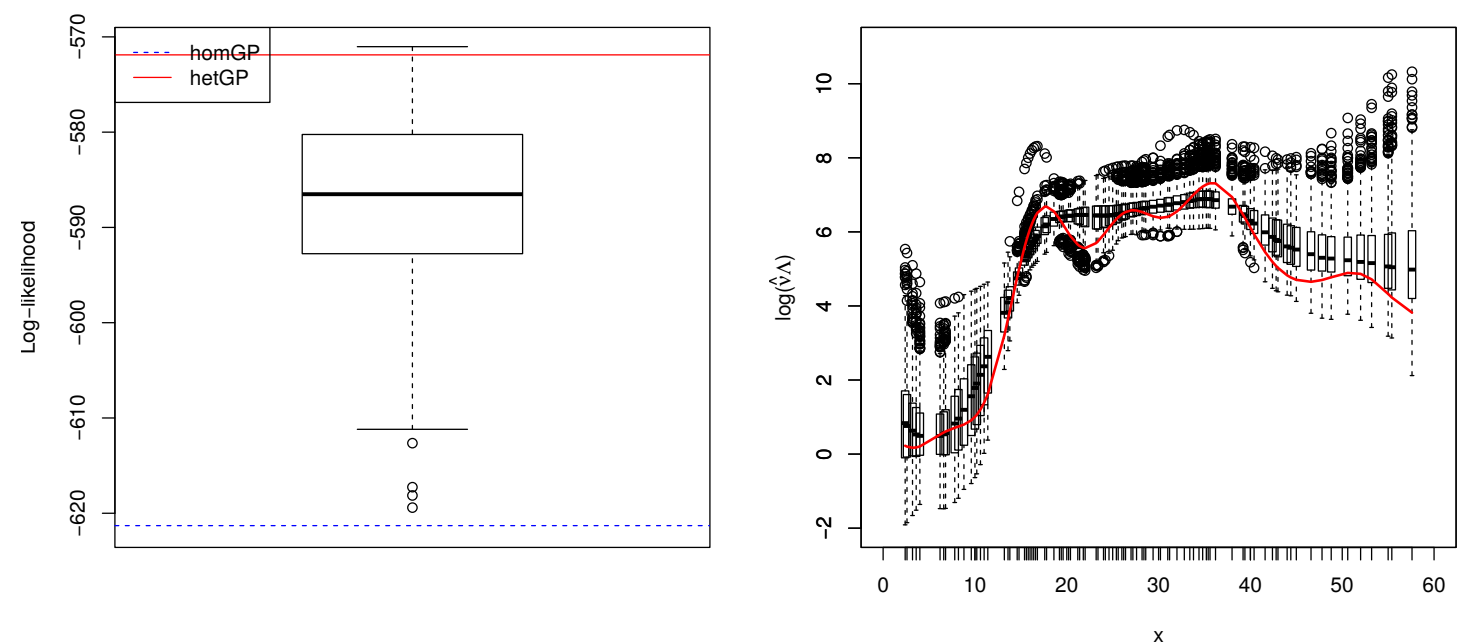

Noisy Branin
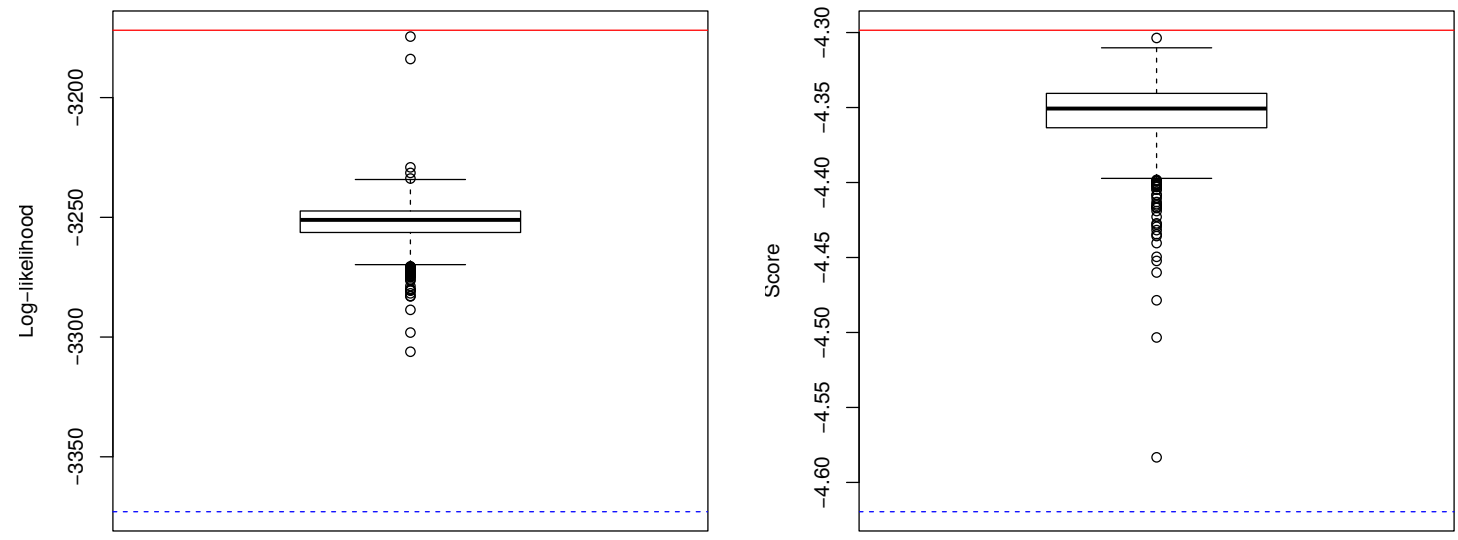

Figure 5: The left column compares joint MLE-values from one thousand random starting points to the proposed starting point, for the motorcycle data (top) and a $2 \mathrm{~d}$ Branin test case (bottom). The top right panel shows boxplots for locally optimal $\log \left(\hat{\nu}_{n} \boldsymbol{\Lambda}_{n}\right)$ values returned for the motorcycle data. The bottom right panel is a boxplot of scores for the 2d Branin example (based on a $51 \times 51$ grid with 10 replicates each as testing data). The performance of hetGP, i.e., under thoughtful initialization, is represented by the solid red in all four panels. A blue dotted line shows the homoskedastic fit for reference.

The results in Figure 5 demonstrate that the proposed optimization procedure is well calibrated. In all four panels, boxplots show the distribution of estimates (via log likelihoods, latent variables, or proper scores, respectively), drawing a comparison to values obtained 
under our thoughtful initialization (in solid red), relative to the baseline homoskedastic fit (blue-dashed). More than $99 \%$ of the time our thoughtful initialization leads to better estimates than under random initialization. The top-right panel, which is in log space, shows that some latent variable estimates obtained under random initialization are quite egregiously over-estimated. It is harder to perform such a visualization in $2 \mathrm{~d}$, so the bottom-right panel shows the more aggregated score metric instead.

\section{Non-stationarity}

Input-dependent noise is one form of non-stationarity amongst many. Perhaps the most commonly addressed form of non-stationary is in the mean, however the recent literature caters to many disparate forms (e.g., Gramacy and Lee, 2008; Ba et al., 2012; Snoek et al., 2014; Gramacy and Apley, 2015; Roininen et al., 2016; Marmin et al., 2017). When there is little data available, distinguishing between non-stationarity in the mean and heteroskedasticity of the noise is hard, but even a mis-specified heteroskedastic-only model may be sufficient (i.e., better than a purely stationary one) for many tasks. We illustrate this on two examples. The first one is an univariate sinusoidal function with a jump and the second is the twodimensional function of Gramacy (2007), described above in Section A.2. The results are shown in Figures 6 and 7 , respectively. In both cases, the estimated noise increases in areas where mean dynamics are most rapidly changing. This leads to improved accuracy, and can facilitate active learning heuristics in sequential design (Gramacy and Lee, 2009). Focusing design effort in regions of high noise can be useful even if the true explanation is rapidly changing signal (not high noise). Nevertheless, when the design increases in the "interesting region", either through further exploration or a larger number of replicates, this behavior disappears and a homogeneous model is returned. Investigations on hybrids of noise and mean heterogeneity represents a promising avenue for future research. 

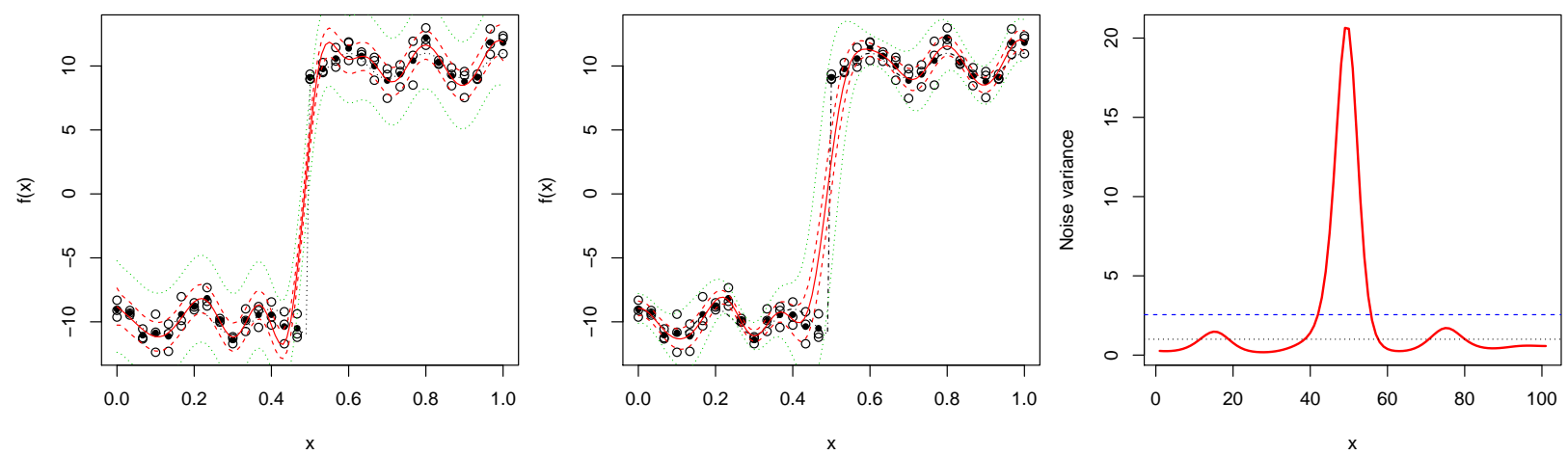

Figure 6: Results from a homoskedastic stationary GP (left) and heteroskedastic stationary GP (center), using the same color code as in Figure 1 based on 31 equidistant points with 3 replicates each. Right: estimated variance with heteroskedastic model (red solid line), with homoskedastic model (blue dashed line) and true unit-variance (black dotted line).
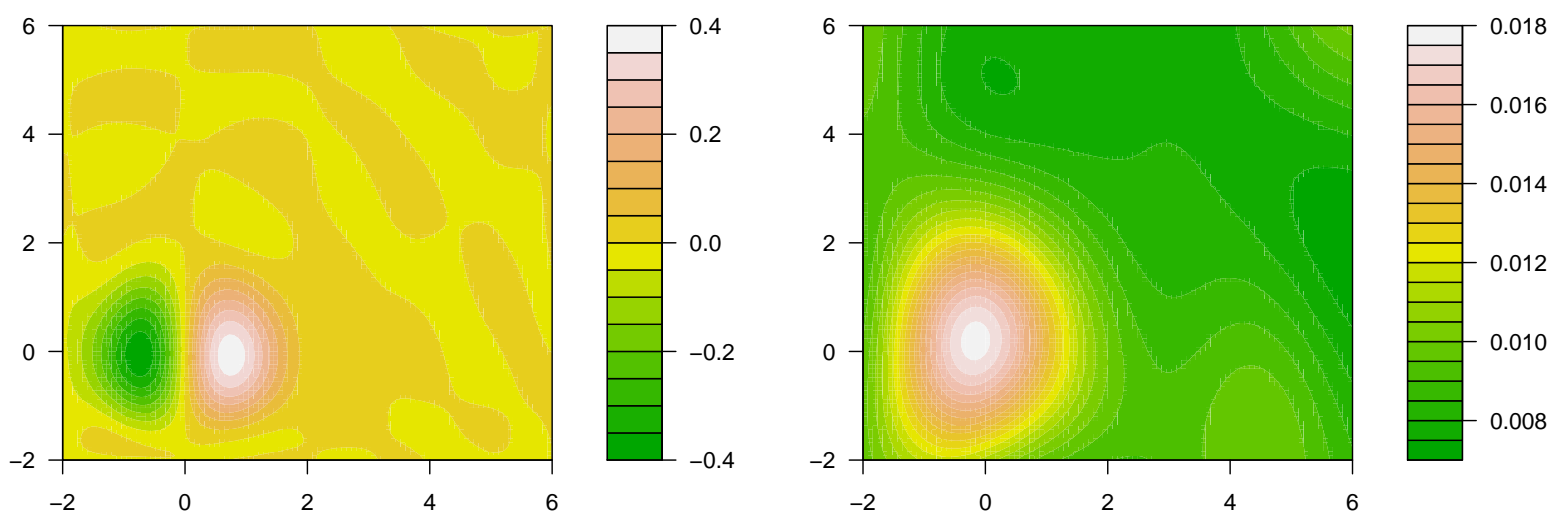

Figure 7: Estimated predictive mean (left) and standard deviation (right) surfaces on the two-bumps function Gramacy (2007), based on a $21 \times 21$ grid with 3 replicates each. The true homogeneous standard deviation used is $10^{-2}$.

\section{References}

Ankenman, B. E., Nelson, B. L., and Staum, J. (2010). "Stochastic kriging for simulation metamodeling." Operations Research, 58, 371-382.

Ba, S., Joseph, V. R., et al. (2012). "Composite Gaussian process models for emulating expensive functions." The Annals of Applied Statistics, 6, 4, 1838-1860.

Banerjee, S., Gelfand, A. E., Finley, A. O., and Sang, H. (2008). "Gaussian predictive 
process models for large spatial data sets." Journal of the Royal Statistical Society: Series B (Statistical Methodology), 70, 4, 825-848.

Binois, M. and Gramacy, R. B. (2017). hetGP: Heteroskedastic Gaussian Process Modeling and Design under Replication. R package version 1.0.0.

Boukouvalas, A. and Cornford, D. (2009). "Learning heteroscedastic Gaussian processes for complex datasets." Tech. rep., Aston University, Neural Computing Research Group.

Core Team, R. (2014). R: A Language And Environment For Statistical Computing. R Foundation for Statistical Computing, Vienna, Austria.

Dixon, L. and Szego, G. (1978). "The global optimization problem: an introduction." Towards global optimization, 2, 1-15.

Eidsvik, J., Shaby, B. A., Reich, B. J., Wheeler, M., and Niemi, J. (2014). "Estimation and prediction in spatial models with block composite likelihoods." Journal of Computational and Graphical Statistics, 23, 295-315.

Erickson, C. B., Ankenman, B. E., and Sanchez, S. M. (2017). "Comparison of Gaussian process modeling software." European Journal of Operational Research.

Gneiting, T. and Raftery, A. E. (2007). "Strictly proper scoring rules, prediction, and estimation." Journal of the American Statistical Association, 102, 477, 359-378.

Goldberg, P. W., Williams, C. K., and Bishop, C. M. (1998). "Regression with inputdependent noise: A Gaussian process treatment." In Advances in Neural Information Processing Systems, vol. 10, 493-499. Cambridge, MA: MIT press.

Gramacy, R. and Lee, H. (2009). "Adaptive Design and Analysis of Supercomputer Experiment." Technometrics, 51, 2, 130-145. 
Gramacy, R. B. (2007). "tgp: an R package for Bayesian nonstationary, semiparametric nonlinear regression and design by treed Gaussian process models." Journal of Statistical Software, 19, 9, 6 .

Gramacy, R. B. and Apley, D. W. (2015). "Local Gaussian process approximation for large computer experiments." Journal of Computational and Graphical Statistics, 24, 2, 561578.

Gramacy, R. B. and Lee, H. K. H. (2008). "Bayesian Treed Gaussian Process Models With an Application to Computer Modeling." Journal of the American Statistical Association, 103, 483, 1119-1130.

Haaland, B. and Qian, P. (2011). "Accurate emulators for large-scale computer experiments." Annals of Statistics, 39, 6, 2974-3002.

Harville, D. (1997). Matrix algebra from a statistician's perspective. Springer-Verlag.

Hong, L. and Nelson, B. (2006). "Discrete optimization via simulation using COMPASS." Operations Research, 54, 1, 115-129.

Hu, R. and Ludkovski, M. (2017). "Sequential design for ranking response surfaces." SIAM/ASA Journal on Uncertainty Quantification, 5, 1, 212-239.

Kamiński, B. (2015). "A method for the updating of stochastic Kriging metamodels." European Journal of Operational Research, 247, 3, 859-866.

Kaufman, C., Bingham, D., Habib, S., Heitmann, K., and Frieman, J. (2012). "Efficient emulators of computer experiments using compactly supported correlation functions, with an application to cosmology." Annals of Applied Statistics, 5, 4, 2470-2492. 
Kersting, K., Plagemann, C., Pfaff, P., and Burgard, W. (2007). "Most likely heteroscedastic Gaussian process regression." In Proceedings of the International Conference on Machine Learning, 393-400. New York, NY: ACM.

Lazaro-Gredilla, M. and Titsias, M. (2011). "Variational heteroscedastic Gaussian process regression." In Proceedings of the International Conference on Machine Learning, 841-848. New York, NY: ACM.

Lee, M. R. and Owen, A. B. (2015). "Single Nugget Kriging." Tech. rep., Stanford University. ArXiv:1507.05128.

Ludkovski, M. and Niemi, J. (2010). "Optimal dynamic policies for influenza management." Statistical Communications in Infectious Diseases, 2, 1, article 5.

Marmin, S., Ginsbourger, D., Baccou, J., and Liandrat, J. (2017). "Warped Gaussian processes and derivative-based sequential design for functions with heterogeneous variations."

Merl, D., Johnson, L. R., Gramacy, R. B., and Mangel, M. (2009). "A statistical framework for the adaptive management of epidemiological interventions." PloS One, 4, 6, e5807.

Ng, S. H. and Yin, J. (2012). "Bayesian kriging analysis and design for stochastic systems." ACM Transations on Modeling and Computer Simulation (TOMACS), 22, 3, article no. 17.

Nychka, D., Bandyopadhyay, S., Hammerling, D., Lindgren, F., and Sain, S. (2015). "A Multi-resolution Gaussian process model for the analysis of large spatial data sets." Journal of Computational and Graphical Statistics, 24, 2, 579-599.

Opsomer, J., Ruppert, D., Wand, W., Holst, U., and Hossler, O. (1999). "Kriging with nonparameteric variance function estimation." Biometrics, 55, 704-710. 
Picheny, V. and Ginsbourger, D. (2013). "A nonstationary space-time Gaussian process model for partially converged simulations." SIAM/ASA Journal on Uncertainty Quantification, 1, 57-78.

Plumlee, M. (2014). "Efficient inference for random fields using sparse grid designs." Journal of the American Statistical Association, 109, 508, 1581-1591.

Quadrianto, N., Kersting, K., Reid, M., Caetano, T., and Buntine, W. (2009). "Kernel conditional quantile estimation via reduction revisited." In Proceedings of the 9th IEEE International Conference on Data Mining, 938-943.

Roininen, L., Girolami, M., Lasanen, S., and Markkanen, M. (2016). "Hyperpriors for Matérn fields with applications in Bayesian inversion." arXiv preprint arXiv:1612.02989.

Roustant, O., Ginsbourger, D., and Deville, Y. (2012). "DiceKriging, DiceOptim: Two R Packages for the analysis of computer experiments by kriging-based metamodeling and optimization." Journal of Statistical Software, 51, 1, 1-55.

Snelson, E. and Ghahramani, Z. (2005). "Sparse Gaussian processes using pseudo-inputs." In Advances in Neural Information Processing Systems, 1257-1264.

Snelson, E. and Gharamani, Z. (2006). "Variable noise and dimensionality reduction in Gaussian processes." In Proceedings of the International Conference in Artificial Intelligence. Also see arXiv:1506.04000.

Snoek, J., Swersky, K., Zemel, R. S., and Adams, R. P. (2014). "Input Warping for Bayesian Optimization of Non-stationary Functions." In International Conference on Machine Learning, 1674-1682.

Venables, W. N. and Ripley, B. D. (2002). Modern Applied Statistics with S. 4th ed. New York: Springer. ISBN 0-387-95457-0. 
Wang, W. and Chen, X. (2016). "The effects of estimation of heteroscedasticity on stochastic kriging." In Proceedings of the 2016 Winter Simulation Conference, 326-337. IEEE Press.

Xie, J., Frazier, P., and Chick, S. (2012). "Assemble to Order Simulator." 